\title{
Flavored dark matter beyond Minimal Flavor Violation
}

\author{
Prateek Agrawal, ${ }^{a}$ Monika Blanke $^{b}$ and Katrin Gemmler ${ }^{a}$ \\ ${ }^{a}$ Fermilab, \\ P.O. Box 500, Batavia, IL 60510, U.S.A. \\ ${ }^{b}$ Theory Division, CERN, \\ CH-1211 Geneva 23, Switzerland \\ E-mail: prateek@fnal.gov, monika.blanke@cern.ch, katrin@fnal.gov
}

ABSTRACT: We study the interplay of flavor and dark matter phenomenology for models of flavored dark matter interacting with quarks. We allow an arbitrary flavor structure in the coupling of dark matter with quarks. This coupling is assumed to be the only new source of violation of the Standard Model flavor symmetry extended by a $\mathrm{U}(3)_{\chi}$ associated with the dark matter. We call this ansatz Dark Minimal Flavor Violation (DMFV) and highlight its various implications, including an unbroken discrete symmetry that can stabilize the dark matter. As an illustration we study a Dirac fermionic dark matter $\chi$ which transforms as triplet under $\mathrm{U}(3)_{\chi}$, and is a singlet under the Standard Model. The dark matter couples to right-handed down-type quarks via a colored scalar mediator $\phi$ with a coupling $\lambda$. We identify a number of "flavor-safe" scenarios for the structure of $\lambda$ which are beyond Minimal Flavor Violation. For dark matter and collider phenomenology we focus on the wellmotivated case of $b$-flavored dark matter. The combined flavor and dark matter constraints on the parameter space of $\lambda$ turn out to be interesting intersections of the individual ones. LHC constraints on simplified models of squarks and sbottoms can be adapted to our case, and monojet searches can be relevant if the spectrum is compressed.

Keywords: Beyond Standard Model, Cosmology of Theories beyond the SM, B-Physics, Kaon Physics

ARXiv EPRINT: 1405.6709 


\section{Contents}

1 Introduction $\quad 2$

2 Flavored dark matter beyond MFV - a minimal model 5

3 Implications of the Dark Minimal Flavor Violation hypothesis $\quad 6$

3.1 New flavor violating parameters and a convenient parametrization for $\lambda \quad 6$

$\begin{array}{lll}3.2 & \text { Non-DMFV contributions and dark matter stability } & 8\end{array}$

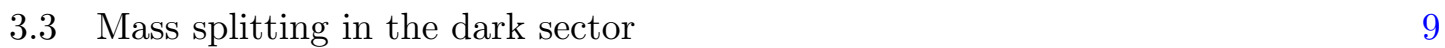

4 Constraints from flavor and precision observables $\quad 9$

$\begin{array}{ll}4.1 \text { Constraints from meson anti-meson mixing } & 10\end{array}$

4.2 Radiative and rare $K$ and $B$ decays $\quad 11$

$\begin{array}{lll}4.3 & \text { Electroweak precision tests and electric dipole moments } & 13\end{array}$

5 Flavor pre-analysis of possible structures for the DM-quark coupling $\quad 13$

$\begin{array}{lll}5.1 & \text { Strategy of the numerical analysis } & 13\end{array}$

$\begin{array}{ll}5.2 \text { "Flavor-safe" scenarios for the structure of } \lambda & 15\end{array}$

$\begin{array}{ll}\text { 5.3 A note on flavor safety of the UV completion } & 17\end{array}$

$\begin{array}{lll}5.4 & \text { Recovering the MFV limit in the structures for } \lambda & 18\end{array}$

6 From the flavor pre-analysis to dark matter scenarios 19

7 Phenomenology of $b$-flavored dark matter $\quad 21$

$\begin{array}{lll}7.1 & \text { Relic abundance } & 21\end{array}$

$\begin{array}{lll}7.2 & \text { Direct detection } & 23\end{array}$

8 Combined numerical analysis of flavor and dark matter constraints $\quad 24$

8.1 Single flavor freeze-out 24

$\begin{array}{ll}8.2 \text { 13-degeneracy } & 27\end{array}$

$\begin{array}{ll}8.3 \text { 23-degeneracy } 29 & 29\end{array}$

$\begin{array}{ll}8.4 \text { Quasi-degenerate dark sector } & 29\end{array}$

9 Collider phenomenology $\quad 30$

9.1 Multijet with missing energy searches 31

$\begin{array}{lll}9.2 & \text { Monojet searches } & 32\end{array}$

$\begin{array}{lll}9.3 & \text { Distinctive signatures } & 35\end{array}$

10 Conclusions $\quad 35$

$\begin{array}{ll}\text { A Classification of DMFV symmetry structures } & 37\end{array}$

$\begin{array}{lll}\mathrm{B} & \mathbb{Z}_{3} \text { as an unbroken subgroup of DMFV } & 38\end{array}$

$\begin{array}{ll}\text { C Relevant loop functions } & 39\end{array}$

$\begin{array}{ll}\text { D Decay of heavier flavors } & 39\end{array}$ 


\section{Introduction}

Dark matter (DM) provides a strong connection between the two phenomenologically rich arenas: particle astrophysics and beyond Standard Model (SM) physics. While the existence of DM is part of the standard model of cosmology, its particle physics origins are largely unknown. The WIMP (weakly interacting massive particle) miracle however provides a tantalizing hint that DM is associated with new physics (NP) at the weak scale, and such candidates should be accessible to various ongoing experiments. Signals at these experiments depend strongly on the nature of interactions of the DM with SM fields, and are less sensitive to other details of the model. This motivates the study of simplified models, which minimally extend the SM to include couplings of DM particles with the SM. Each simplified model can then capture the dark matter phenomenology of a wide range of models.

Once we consider different classes of simplified models, one new category of models arises in analogy with SM flavor: flavored DM [1-9]. In this setup DM particles come in multiple copies, and have a non-trivial flavor structure in their couplings with quarks and leptons. ${ }^{1}$ This framework does show up in a very specific way in supersymmetric models as sneutrino DM models [12-18], but clearly there are more general possibilities.

This class of models is constrained, like other DM models, by both indirect and direct detection DM experiments as well as collider searches. The relevant schematic interaction responsible for these signatures is shown in the left panel of figure 1. Additionally precision flavor experiments have to be taken into account due to the flavor violation introduced by the dark sector. Schematically this contribution is displayed in the right panel of figure 1, adding a new class of diagrams to the well-studied DM-SM interaction.

Flavored dark matter models can have significantly distinct phenomenology. For indirect detection experiments, the spectrum of photons and leptons arising from DM annihilation depends on the relative annihilation into various final states. For example, it was shown that a DM candidate annihilating exclusively to $b$-quarks provides a good fit to the spectrum of excess photons observed in a recent analysis of Fermi-LAT data from the galactic center [19-33]. Direct detection predictions for scattering vary widely depending upon whether the ambient DM particles couple to the first generation quarks directly or not. The absence of direct detection signals so far then point to the possibility of suppression of such a coupling, which can be achieved through either a loop suppression or a small mixing angle. Collider searches for DM are also sensitive to the DM couplings to various quark flavors, both in terms of the DM production cross section, as well as the flavor pattern of visible final states which can be produced in association with the DM.

While some of these effects have been explored, the study of flavor phenomenology has largely been restricted to elaborate models such as the MSSM. Previous analyses often assume for simplicity universality or minimal flavor violation (MFV) [34-38], so that flavor changing neutral current (FCNC) effects are automatically suppressed. On one hand this

\footnotetext{
${ }^{1}$ An alternative scenario in which dark matter arises from a discrete $A_{4}$ symmetry in the neutrino sector has been considered in [10, 11].
} 

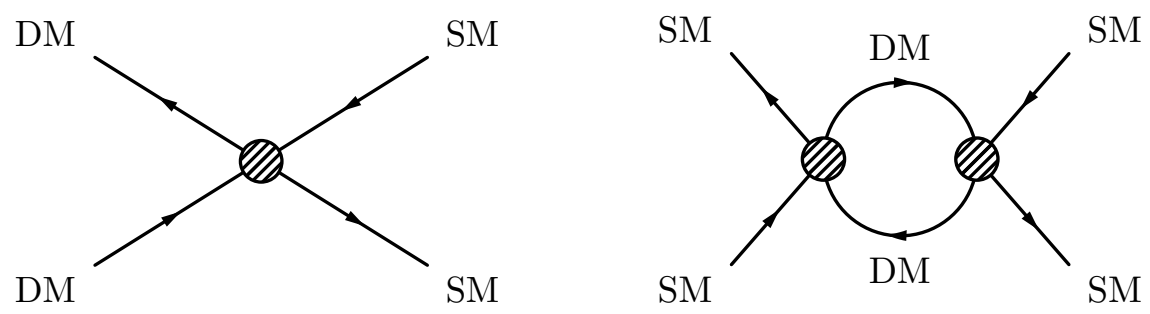

Figure 1. Schematic diagrams contributing to experimental constraints on flavored DM.

is welcome due to the good agreement of the flavor data with the SM prediction, but on the other hand interesting effects in the flavor sector are eliminated.

In this paper we abandon the MFV principle and consider instead a general flavor violating coupling of DM particles with quarks. DM is introduced as a triplet under a new global flavor symmetry $\mathrm{U}(3)_{\chi}$. While in our analysis the coupling matrix (denoted by $\lambda$ ) is taken to be completely general, we make one simplifying assumption that turns out to be helpful in various respects. We impose that $\lambda$ is the only new source of flavor breaking, in addition to the SM Yukawa couplings. As this assumption generalizes the MFV principle to the DM sector, we call it Dark Minimal Flavor Violation (DMFV). We will point out the following features of DMFV:

- The DMFV framework, while bearing some conceptual similarity to MFV, goes well beyond the latter framework, as it allows for large FCNC effects. The structure of $\lambda$ needs to be determined from the available constraints.

- The DMFV ansatz naturally preserves a residual $\mathbb{Z}_{3}$ symmetry, which guarantees the stability of the DM particle.

- DMFV significantly reduces the number of new parameters in the Lagrangian, as the DM mass term $m_{\chi}$ must be flavor conserving up to corrections of the form $\lambda^{\dagger} \lambda$.

- DMFV guarantees "flavor-safety" of the UV complete theory. It is therefore sufficient to identify flavor-safe scenarios for the structure of $\lambda$ within the simplified model framework.

In the phenomenological part of our paper we will restrict ourselves to the study of the simplest version of DMFV, which we refer to as the minimal DMFV (mDMFV) model in order to distinguish it from the more general framework. The DM is taken to be a Dirac fermion $\chi$, interacting with the right-handed down-type quarks via the coupling

$$
\lambda \bar{d}_{R} \chi \phi
$$

with a scalar mediator $\phi$. While leaving the question of a possible UV completion unanswered, this study captures the most important phenomenological effects accessible to current experiments. Our studies extend the existing literature on the phenomenology of flavored DM in the following ways: 
- We go beyond the simple MFV hypothesis that automatically suppresses all flavor effects to an acceptable level. Instead we study the implications of a completely general coupling matrix $\lambda$, embedded in the DMFV ansatz, and derive its structure from the experimental constraints.

- We consider a large number of relevant precision observables which can potentially be affected by the mDMFV model. These are in particular the constraints from meson-antimeson mixing, radiative and rare $B$ and $K$ decays, electroweak precision observables and electric dipole moments.

- From the analysis of meson-antimeson mixing observables we identify a number of "flavor-safe" scenarios for the structure of $\lambda$. These scenarios will be useful for future studies of flavored DM models beyond MFV, as they can be imposed simply and render detailed re-analyses unnecessary.

- Subsequently we perform a simultaneous analysis of flavor and DM constraints, such as the relic abundance from thermal freeze-out, and direct detection data from LUX [39]. While restricting ourselves to the phenomenologically interesting case of $b$-flavored DM, we consider several mass hierarchies in the dark sector, i. e. large and small splittings between the DM particle and the heavier flavors.

- We reveal a non-trivial interplay of the complementary flavor and DM constraints, such that the combined constraint on the parameter space of $\lambda$ turns out to be interesting intersections of the individual ones. This result underlines the importance of taking into account the various constraints simultaneously.

- We point out a cancellation between various mDMFV one-loop contributions (photon penguin and box diagram) to the WIMP-nucleon scattering, occurring for a certain range of coupling parameters. As the photon penguin is only present for scattering off protons, while the box diagram contributes to proton and neutron scattering crosssections, this cancellation provides a possible realization of Xenophobic DM [40, 41].

- We review the constraints from collider searches on the mDMFV model with $b$ flavored DM. The most stringent bounds are placed by searches for bottom squark pair production, constraining the parameter space of the model up to a mediator mass $m_{\phi} \sim 800-900 \mathrm{GeV}$. Monojet searches can be important for very compressed spectra, or for very heavy $\phi$ such that its direct production is suppressed.

Our paper is organized as follows. In section 2 we introduce the concept of Dark Minimal Flavor Violation (DMFV), and describe the minimal model realizing this hypothesis, the mDMFV model. Section 3 deals with the implications of the DMFV hypothesis that are valid beyond the minimal model. In section 4 we provide the formalism for a detailed study of the constraints from meson anti-meson mixing on the mDMFV model. We also consider potential new contributions to radiative and rare $B$ and $K$ decays, electroweak precision observables and electric dipole moments and find all of these observables to be 
SM-like. Section 5 is devoted to a detailed numerical analysis of the constraints on the coupling matrix $\lambda$ arising from meson-antimeson mixing. We identify a number of "flavor-safe" scenarios for the coupling matrix $\lambda$. In section 6 we provide a comprehensive summary of the results of the numerical flavor analysis and the different scenarios emerging for the analysis of DM constraints. In section 7 we study the DM phenomenology of the mDMFV model, considering both the relic abundance constraint from thermal freeze-out and the emerging WIMP-nucleon cross section observed in direct detection experiments. A combined numerical analysis of flavor and DM constraints is performed in section 8, studying the various possible mass hierarchies in turn. In section 9 we estimate the constraints on the mDMFV model from the LHC, stemming in particular from monojet searches and searches for supersymmetric bottom squarks. We also mention some new signatures for these models. In section 10 we summarize our results. Some technical details are relegated to the appendices.

\section{Flavored dark matter beyond MFV — a minimal model}

We consider a setup where DM $\chi$ transforms in the fundamental representation of a new flavor symmetry $\mathrm{U}(3)_{\chi}$, in analogy with the SM flavor symmetry. While we posit this symmetry as an ansatz, it will be an interesting future direction to study possible UV completions (e.g. extended Grand Unified Theories) which incorporate this structure.

We assume that the global

$$
\mathrm{U}(3)_{q} \times \mathrm{U}(3)_{u} \times \mathrm{U}(3)_{d} \times \mathrm{U}(3)_{\chi}
$$

flavor symmetry is broken only by the SM Yukawa couplings $Y_{u}, Y_{d}$ and the DM-quark coupling $\lambda$. This ansatz generalizes the MFV hypothesis [34-38] to include an extra $\mathrm{U}(3)_{\chi}$ symmetry under which the DM field transforms, and an additional Yukawa coupling $\lambda$. We refer to this assumption as Dark Minimal Flavor Violation (DMFV). Depending on the type of quark to which the DM couples, different classes of DMFV can be defined, see appendix A for details.

In what follows we restrict ourselves to the coupling of $\chi$ to right-handed down-type quarks via a scalar mediator $\phi$. While the DM particle $\chi$ is a gauge singlet, the mediator $\phi$ has to carry color and hypercharge. This helps to keep the model simple, since no further electroweak structure is required when assuming the new particles to be singlets under $\mathrm{SU}(2)_{L}$. Further the choice of down-type quarks ensures to have an effect in relevant flavor observables such as $K$ and $B_{d, s}$ meson mixing and well-measured rare decays.

The most general renormalizable Lagrangian including the minimal field content is then given by

$$
\begin{aligned}
\mathcal{L}= & \mathcal{L}_{\mathrm{SM}}+i \bar{\chi} \not \partial \chi-m_{\chi} \bar{\chi} \chi-\left(\lambda_{i j} \bar{d}_{R i} \chi_{j} \phi+\text { h.c. }\right) \\
& +\left(D_{\mu} \phi\right)^{\dagger}\left(D^{\mu} \phi\right)-m_{\phi}^{2} \phi^{\dagger} \phi+\lambda_{H \phi} \phi^{\dagger} \phi H^{\dagger} H+\lambda_{\phi \phi} \phi^{\dagger} \phi \phi^{\dagger} \phi,
\end{aligned}
$$

with the symmetry transformation properties summarized in table 1 . Note that the $\mathrm{U}(3)_{\chi}$ flavor symmetry in the DM sector guarantees that at the Lagrangian level all three DM 


\begin{tabular}{|c|ccc|cccc|}
\hline & $\mathrm{SU}(3)_{c}$ & $\mathrm{SU}(2)_{L}$ & $\mathrm{U}(1)_{Y}$ & $\mathrm{U}(3)_{q}$ & $\mathrm{U}(3)_{u}$ & $\mathrm{U}(3)_{d}$ & $\mathrm{U}(3)_{\chi}$ \\
\hline$q_{L}$ & $\mathbf{3}$ & $\mathbf{2}$ & $1 / 6$ & $\mathbf{3}$ & $\mathbf{1}$ & $\mathbf{1}$ & $\mathbf{1}$ \\
$u_{R}$ & $\mathbf{3}$ & $\mathbf{1}$ & $2 / 3$ & $\mathbf{1}$ & $\mathbf{3}$ & $\mathbf{1}$ & $\mathbf{1}$ \\
$d_{R}$ & $\mathbf{3}$ & $\mathbf{1}$ & $-1 / 3$ & $\mathbf{1}$ & $\mathbf{1}$ & $\mathbf{3}$ & $\mathbf{1}$ \\
\hline$\ell_{L}$ & $\mathbf{1}$ & $\mathbf{2}$ & $-1 / 2$ & $\mathbf{1}$ & $\mathbf{1}$ & $\mathbf{1}$ & $\mathbf{1}$ \\
$e_{R}$ & $\mathbf{1}$ & $\mathbf{1}$ & -1 & $\mathbf{1}$ & $\mathbf{1}$ & $\mathbf{1}$ & $\mathbf{1}$ \\
\hline$H$ & $\mathbf{1}$ & $\mathbf{2}$ & $1 / 2$ & $\mathbf{1}$ & $\mathbf{1}$ & $\mathbf{1}$ & $\mathbf{1}$ \\
\hline$\phi$ & $\mathbf{3}$ & $\mathbf{1}$ & $-1 / 3$ & $\mathbf{1}$ & $\mathbf{1}$ & $\mathbf{1}$ & $\mathbf{1}$ \\
$\chi_{L}$ & $\mathbf{1}$ & $\mathbf{1}$ & 0 & $\mathbf{1}$ & $\mathbf{1}$ & $\mathbf{1}$ & $\mathbf{3}$ \\
$\chi_{R}$ & $\mathbf{1}$ & $\mathbf{1}$ & 0 & $\mathbf{1}$ & $\mathbf{1}$ & $\mathbf{1}$ & $\mathbf{3}$ \\
\hline$Y_{u}$ & $\mathbf{1}$ & $\mathbf{1}$ & 0 & $\mathbf{3}$ & $\overline{\mathbf{3}}$ & $\mathbf{1}$ & $\mathbf{1}$ \\
$Y_{d}$ & $\mathbf{1}$ & $\mathbf{1}$ & 0 & $\mathbf{3}$ & $\mathbf{1}$ & $\overline{\mathbf{3}}$ & $\mathbf{1}$ \\
$\lambda$ & $\mathbf{1}$ & $\mathbf{1}$ & 0 & $\mathbf{1}$ & $\mathbf{1}$ & $\mathbf{3}$ & $\overline{\mathbf{3}}$ \\
\hline
\end{tabular}

Table 1. Symmetry transformation properties of the minimal DMFV matter content and the Yukawa spurions.

flavors have the same mass $m_{\chi}$, although they acquire a small splitting from higher order DMFV corrections. In what follows we refer to this model as the minimal DMFV (mDMFV) model.

The mDMFV model has some similarities to simplified models of supersymmetry and should be understood in an analogous manner. In contrast to the SUSY case however in mDMFV the flavor charge is carried by the DM fermions and not by the scalar mediator. Further we assume $\chi$ to be a Dirac fermion (a Majorana mass term would violate the $\mathrm{U}(3)_{\chi}$ symmetry), while in the minimal SUSY models the gauginos are Majorana.

We stress that the DMFV ansatz, in contrast to the MFV ansatz, potentially allows for large flavor violating effects. A careful analysis of FCNC constraints is therefore necessary.

\section{Implications of the Dark Minimal Flavor Violation hypothesis}

In the present section we consider the consequences of the DMFV ansatz. We stress that these implications go beyond the simple mDMFV model introduced in section 2 and hold in any scenario with the same DMFV flavor symmetry breaking pattern.

\subsection{New flavor violating parameters and a convenient parametrization for $\lambda$}

In the DMFV setup the flavor symmetry in the quark sector is broken only by the SM Yukawa couplings $Y_{u}, Y_{d}$ and the DM-quark coupling $\lambda$. In a first step the SM flavor symmetry can be used to remove unphysical parameters from the SM Yukawas. They can be parametrized as usual in terms of the six quark masses and the CKM matrix, signaling the misalignment between $Y_{u}$ and $Y_{d}$. 
In the second step we remove unphysical parameters from the coupling matrix $\lambda$. Being an arbitrary complex matrix, it contains at first 9 real parameters and 9 complex phases. Some of them can be removed by making use of the DM flavor symmetry $\mathrm{U}(3)_{\chi}$.

We start by parametrizing $\lambda$ in terms of a singular value decomposition

$$
\lambda=U_{\lambda} D_{\lambda} V_{\lambda}
$$

where $D_{\lambda}$ is a diagonal matrix with real and positive entries, and $U_{\lambda}$ and $V_{\lambda}$ are unitary matrices. Note that $U_{\lambda}$ and $V_{\lambda}$ are not uniquely defined, as $\lambda$ is invariant under the diagonal rephasing

$$
U_{\lambda}^{\prime}=U_{\lambda} \operatorname{diag}\left(e^{i \theta_{1}}, e^{i \theta_{2}}, e^{i \theta_{3}}\right), \quad V_{\lambda}^{\prime}=\operatorname{diag}\left(e^{-i \theta_{1}}, e^{-i \theta_{2}}, e^{-i \theta_{3}}\right) V_{\lambda}
$$

We use this freedom to reduce the number of phases in $U_{\lambda}$ to three. Then $\lambda$ has 9 real parameters and 9 phases in the parametrization (3.1). We can now use the $\mathrm{U}(3)_{\chi}$ invariance to fully remove the unitary matrix $V_{\lambda}$. Consequently we are left with the matrix

$$
\lambda=U_{\lambda} D_{\lambda}
$$

It contains nine parameters: three non-negative elements of $D_{\lambda}$, and three mixing angles and three $\mathrm{CP}$ violating phases in $U_{\lambda}$. Note that the mixing angles are restricted to the range $0 \leq \theta_{i j}^{\lambda} \leq \pi / 4$ in order to avoid a double-counting of parameter space. This choice ensures that each DM flavor couples dominantly to the quark of the same generation. For instance we can refer to $\chi_{3}$ as $b$-flavored DM.

A convenient parametrization for $U_{\lambda}$ has been derived in [42] in the context of the Littlest Higgs model with T-parity. It can be written as

$$
\begin{aligned}
U_{\lambda} & =U_{23}^{\lambda} U_{13}^{\lambda} U_{12}^{\lambda} \\
& =\left(\begin{array}{ccc}
1 & 0 & 0 \\
0 & c_{23}^{\lambda} & s_{23}^{\lambda} e^{-i \delta_{23}^{\lambda}} \\
0-s_{23}^{\lambda} e^{i \delta_{23}^{\lambda}} & c_{23}^{\lambda}
\end{array}\right)\left(\begin{array}{ccc}
c_{13}^{\lambda} & 0 & s_{13}^{\lambda} e^{-i \delta_{13}^{\lambda}} \\
0 & 1 & 0 \\
-s_{13}^{\lambda} e^{i \delta_{13}^{\lambda}} & 0 & c_{13}^{\lambda}
\end{array}\right)\left(\begin{array}{ccc}
c_{12}^{\lambda} & s_{12}^{\lambda} e^{-i \delta_{12}^{\lambda}} & 0 \\
-s_{12}^{\lambda} e^{i \delta_{12}^{\lambda}} & c_{12}^{\lambda} & 0 \\
0 & 0 & 1
\end{array}\right),
\end{aligned}
$$

where $c_{i j}^{\lambda}=\cos \theta_{i j}^{\lambda}$ and $s_{i j}^{\lambda}=\sin \theta_{i j}^{\lambda}$. Performing the product one obtains the expression

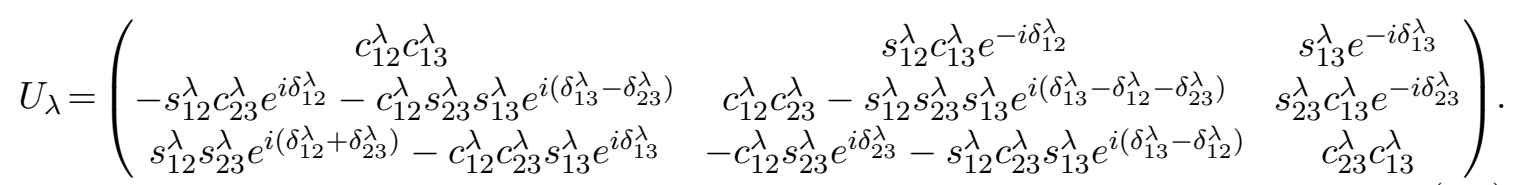

Finally it turns out to be convenient to parametrize the diagonal matrix $D_{\lambda}$ as

$$
D_{\lambda} \equiv \operatorname{diag}\left(D_{\lambda, 11}, D_{\lambda, 22}, D_{\lambda, 33}\right)=\lambda_{0} \cdot \mathbb{1}+\operatorname{diag}\left(\lambda_{1}, \lambda_{2},-\left(\lambda_{1}+\lambda_{2}\right)\right) .
$$

The first parametrization is useful for the analysis of DM and collider constraints. The second parametrization instead is better suited for the flavor analysis, since it quantifies the deviations from a flavor universal coupling. 


\subsection{Non-DMFV contributions and dark matter stability}

The flavor structure of the SM is accidental - there exist no other gauge-invariant operators beyond the Yukawa terms at the renormalizable level. It is then worth asking if the DMFV ansatz can also arise naturally in an analogous way. In this section we study how generic the DMFV ansatz is from a UV point-of-view. We stress however that the goal of this work is merely to study the novel phenomenology arising from this ansatz, and a complete UV model is beyond the current scope. We merely study the corrections to the ansatz to the extent that they can affect low energy phenomenology, particularly DM decay.

Interestingly, in the exact DMFV limit, all operators inducing decay of the $\mathrm{U}(3)_{\chi}$ triplet $\chi$ are forbidden, even at the non-renormalizable level. In analogy to the stability of DM in the MFV case [3] it can straightforwardly be shown - see appendix B for details - that the flavor symmetry (2.1) broken only by the Yukawa couplings $Y_{u}, Y_{d}$ and $\lambda$, together with $\mathrm{SU}(3)_{\mathrm{QCD}}$ imply an unbroken $\mathbb{Z}_{3}$ symmetry. It is then natural to impose this $\mathbb{Z}_{3}$ symmetry as exact, under which only the new particles $\chi_{i}$ and $\phi$ are charged, and transform as

$$
\chi_{i} \rightarrow e^{2 \pi i / 3} \chi_{i}, \quad \phi \rightarrow e^{-2 \pi i / 3} \phi .
$$

This symmetry prevents the decay of any of these states into SM particles only, and therefore renders the lightest state stable.

We now estimate the size of non-DMFV effects that can arise. We imagine a UV scale, $\Lambda$, above which the DM flavor symmetry $\mathrm{U}(3)_{\chi}$ is unbroken. While this scale could in principle be associated with the SM flavor scale as well, for simplicity we assume that the SM flavor structure is generated at a higher scale. Generically, we expect all operators allowed by symmetries to be generated at the scale $\Lambda$. The most important contributions at low energy arise from relevant and marginal operators.

The relevant operator

$$
\mathcal{O}_{m}=\delta m_{i j} \bar{\chi}_{i} \chi_{j}
$$

is the leading operator that is generated. It maximally violates the DMFV ansatz, while preserving the $\mathbb{Z}_{3}$. This operator can be prevented from being generated at the scale $\Lambda$ if the mass of the DM fermions is generated at a lower scale, through a flavor-blind sector. Note the analogy to flavor-blind SUSY breaking, which yields MFV. It is an open question whether such a scenario can be achieved simply in this framework. We assume henceforth that this operator is negligible.

At the marginal level, we generate the following two operators,

$$
\begin{aligned}
& \mathcal{O}_{L}=\bar{\ell}_{L} \chi H+\text { h.c. } \\
& \mathcal{O}_{B}=\bar{q}_{L}\left(i \sigma^{2}\right) q_{L}^{\dagger} \phi^{\dagger}+\text { h.c., }
\end{aligned}
$$

which are lepton and baryon number violating respectively. These are prohibited by the discrete $\mathbb{Z}_{3}$ symmetry, however. 
We see that additional discrete symmetries can be imposed in order to prevent the DM from decaying, and preventing non-DMFV contributions at the renormalizable level. Higher dimensional non-DMFV operators can then only modify other aspects of phenomenology, which are not as severely constrained. Therefore, for a reasonably high scale $\Lambda$, these operators are not expected to alter the phenomenology appreciably.

\subsection{Mass splitting in the dark sector}

As noted in section 2 the DMFV hypothesis ensures that to leading order in the coupling $\lambda$, the masses for different DM particles are equal. There are three potential sources for splittings.

Firstly, there can be contributions to the mass matrix $m_{\chi}$ directly violating DMFV. We assume that such contributions are absent. Secondly, higher dimensional DMFV-violating contributions can still induce splittings, but these are expected to be suppressed by the heavy scale where DMFV is broken.

An unavoidable contribution is through the renormalization group running, where a universal mass at the high scale is renormalized by the presence of the DM coupling $\lambda$ at low scales. Generically, it is also possible that there is a DMFV preserving contribution $\propto \lambda^{\dagger} \lambda$ at tree level. If present, this would be the largest contribution to the DM splittings. Of course, the pattern of splittings generated by the running and by such threshold effects is identical, since both cases are consistent with DMFV.

The splittings are given by

$$
m_{i j}=m_{\chi}\left(\mathbb{1}+\eta \lambda^{\dagger} \lambda+\cdots\right)_{i j}=m_{\chi}\left(1+\eta\left(D_{\lambda, i i}\right)^{2}+\cdots\right) \delta_{i j},
$$

where summation is not implied in the last term. Here $\eta$ is a real coefficient whose value depends on the details of the model. If the contribution to the mass matrix arises at tree level, then $\eta$ is expected to be an $\mathcal{O}(1)$ number. On the other hand, the contribution from running is schematically given by

$$
\eta \sim \frac{1}{16 \pi^{2}} \log \left(\frac{m_{\chi}^{2}}{\Lambda^{2}}\right),
$$

where $\Lambda$ is the dark flavor scale noted above.

The DMFV expansion above is only valid if higher order corrections are parametrically suppressed. In order to ensure convergence, in what follows we will assume $\left|\eta\left(D_{\lambda, i i}\right)^{2}\right|<0.3$.

\section{Constraints from flavor and precision observables}

In this section we study all relevant constraints from flavor observables on the mDMFV model. We start the analysis of the well-measured and strongly constraining observables from meson anti-meson mixing, followed by relevant rare decays. Finally we take a brief look at electroweak precision tests and electric dipole moments. While we will find that $\Delta F=2$ processes significantly shape the structure of a phenomenologically viable coupling matrix $\lambda$, effects of other flavor observables are negligible. 


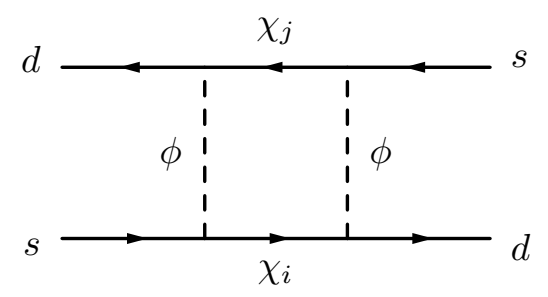

Figure 2. New contribution to $K^{0}-\bar{K}^{0}$ mixing in the mDMFV model.

We restrict ourselves to providing the formulae directly relevant for our study, a more detailed description of relevant techniques and necessary formulae for the study of $\Delta F=2$ processes reaching from effective Hamiltonian to flavor observables can be found for instance in [43]. A recent comprehensive review can be found in [44].

\subsection{Constraints from meson anti-meson mixing}

In the mDMFV model new contributions to $\Delta F=2$ processes arise first at the one loop level. The relevant box diagram is shown in figure 2 for the case of $K^{0}-\bar{K}^{0}$ mixing. Evaluating this diagram we obtain the following contribution to the effective Hamiltonian:

$$
\mathcal{H}_{\mathrm{eff}}^{\Delta S=2, \text { new }}=\frac{1}{128 \pi^{2} m_{\phi}^{2}} \sum_{i, j} \lambda_{s i} \lambda_{d i}^{*} \lambda_{s j} \lambda_{d j}^{*} F\left(x_{i}, x_{j}\right) \times Q^{V R R}+\text { h.c. },
$$

with $x_{i}=m_{\chi_{i}}^{2} / m_{\phi}^{2}$, and the loop function $F\left(x_{i}, x_{j}\right)$ can be found in appendix C. As the new particles $\phi$ and $\chi_{i}$ couple only to right-handed down-type quarks, the only effective operator which receives new contributions is

$$
Q^{V R R}=\left(\bar{s}_{\alpha} \gamma^{\mu} P_{R} d_{\alpha}\right)\left(\bar{s}_{\beta} \gamma_{\mu} P_{R} d_{\beta}\right)
$$

i. e. the chirality-flipped counterpart of the SM operator.

The mass splittings among the $\chi_{i}$ fields constitutes a higher order correction in the DMFV expansion, which we assume to be small. Thus we can take the limit of equal $\chi$ masses in (4.1). The effective Hamiltonian then simplifies to

$$
\mathcal{H}_{\text {eff }}^{\Delta S=2, \text { new }}=\frac{1}{128 \pi^{2} m_{\phi}^{2}} F(x) \xi_{K}^{2} \times Q^{V R R}+\text { h.c. },
$$

where $x=m_{\chi}^{2} / m_{\phi}^{2}$. The loop function $F(x)$ can be found in appendix C. We also defined

$$
\xi_{K}=\left(\lambda \lambda^{\dagger}\right)_{s d}=\sum_{i=1}^{3} \lambda_{s i} \lambda_{d i}^{*} .
$$

The mDMFV contribution of the DM sector to the off-diagonal element of the $K^{0}-\bar{K}^{0}$ mass matrix can then be obtained from

$$
M_{12}^{K, \text { new }}=\frac{1}{2 m_{K}}\left\langle\bar{K}^{0}\left|\mathcal{H}_{\text {eff }}^{\Delta S=2 \text {,new }}\right| K^{0}\right\rangle^{*} .
$$


Using

$$
\left\langle Q^{V R R}(\mu=2 \mathrm{GeV})\right\rangle=\frac{2}{3} m_{K}^{2} F_{K}^{2} \hat{B}_{K}
$$

we obtain

$$
M_{12}^{K, \text { new }}=\frac{1}{384 \pi^{2} m_{\phi}^{2}} m_{K} F_{K}^{2} \hat{B}_{K} \eta_{2} F(x)\left(\xi_{K}^{*}\right)^{2} .
$$

The parameter $\eta_{2}$ summarizes the corrections from the renormalization group running from the weak scale $\mu \sim m_{t}$ down to the scale $\mu=2 \mathrm{GeV}$, where the lattice calculations are performed, as well as the corrections due to the matching of the full theory to the effective theory calculated within the SM.

By parametrizing the NLO corrections by $\eta_{2}$, we make two approximations. We neglect the running from the NP scale $\mu \sim m_{\phi}$ to the scale $\mu \sim m_{t}$, as well as the difference in the matching conditions between the SM and the NP scenario studied here. In order to estimate the error associated to our approach, it is useful to compare our case with the discussion of the 331 models in [45]. In the latter framework the inclusion of the nextto-leading order (NLO) corrections amounts to a few percent correction to the size of the NP contribution. We expect similar conclusions to hold also in our case, in particular since in the MSSM the NLO corrections to the Wilson coefficient $C^{V R R}$ have been found to be small [46].

In an analogous manner we find

$$
M_{12}^{q \text {,new }}=\frac{1}{384 \pi^{2} m_{\phi}^{2}} m_{B_{q}} F_{B_{q}}^{2} \hat{B}_{B_{q}} \eta_{B} F(x)\left(\xi_{B_{q}}^{*}\right)^{2} \quad(q=d, s),
$$

where we define

$$
\xi_{B_{q}}=\left(\lambda \lambda^{\dagger}\right)_{b q}=\sum_{i=1}^{3} \lambda_{b i} \lambda_{q i}^{*} \quad(q=d, s) .
$$

In passing we note that the mDMFV model, coupling only to down-type quarks, does not contribute to $D$ meson observables at the one loop level.

\subsection{Radiative and rare $K$ and $B$ decays}

We now turn our attention to radiative and rare decays, starting with the electromagnetic dipole operator generating the $b \rightarrow s \gamma$ transition.

The effective Hamiltonian describing the $B \rightarrow X_{s} \gamma$ decay can be written as

$$
\mathcal{H}_{\mathrm{eff}}=\frac{4 G_{F}}{\sqrt{2}} V_{t s}^{*} V_{t b}\left(C_{7} Q_{7}+C_{7}^{\prime} Q_{7}^{\prime}+\cdots\right),
$$

where we omitted the tree level and chromomagnetic dipole operators that contribute to $b \rightarrow s \gamma$ via renormalization group mixing. We use the normalization

$$
\begin{aligned}
Q_{7} & =\frac{e}{16 \pi^{2}} m_{b} \bar{s}_{L} \sigma^{\mu \nu} b_{R} F_{\mu \nu}, \\
Q_{7}^{\prime} & =\frac{e}{16 \pi^{2}} m_{b} \bar{s}_{R} \sigma^{\mu \nu} b_{L} F_{\mu \nu} .
\end{aligned}
$$


In the SM the Wilson coefficient $C_{7}^{\prime}$ is strongly suppressed due to the chiral structure of weak interactions:

$$
C_{7, \mathrm{SM}}^{\prime}=\frac{m_{s}}{m_{b}} C_{7, \mathrm{SM}} .
$$

Conversely the DM in our scenario couples only to right-handed SM fermions - therefore the only relevant new contribution arises in the chirality-flipped Wilson coefficient $C_{7}^{\prime}$. The relevant diagrams are analogous to the ones depicting the gluino contribution in supersymmetric models, replacing the gluino by the DM particles $\chi_{i}$ and the squarks by the scalar mediator $\phi$, and keeping only the coupling to right-handed SM quarks. Correcting for the different coupling and taking into account that $\chi_{i}$ are QCD singlets while the gluino is a color octet, we can straightforwardly obtain the result for $\delta C_{7}^{\prime}$ from [47, 48]. Adjusting eq. (A.5) of [48] to our model, we find

$$
\delta C_{7}^{\prime}=-\frac{1}{6 g_{2}^{2} V_{t s}^{*} V_{t b}} \frac{m_{W}^{2}}{m_{\phi}^{2}} \xi_{B_{s}}^{*} g\left(x_{i}\right),
$$

where the short-hand notation for the relevant combination of elements of $\lambda$ has been defined in (4.9), and the loop function $g(x)$ is given in appendix C.

With $g(x) \sim 0.08-0.17$ the size of the new contribution $\delta C_{7}^{\prime}$ can be estimated as

$$
\left|\delta C_{7}^{\prime}\right| \lesssim 4 \cdot 10^{-2}\left|\xi_{B_{s}}^{*}\right|\left[\frac{500 \mathrm{GeV}}{m_{\phi}}\right]^{2} .
$$

Comparing this result to the constraints on the size of NP contributions, see e. g. figure 2 in [49], we see that the effect on the electromagnetic dipole operators generated in the present model is completely negligible. This is very welcome in view of the good agreement of $\operatorname{Br}\left(B \rightarrow X_{s} \gamma\right)$ with the data.

Contributions to the four-fermion operators mediating transitions like $b \rightarrow s \mu^{+} \mu^{-}$or $s \rightarrow d \nu \bar{\nu}$ can generally be split into tree level and one loop box and penguin diagrams. In the mDMFV model new tree level diagrams are forbidden by the residual $\mathbb{Z}_{3}$ symmetry (see appendix B), while box diagrams are not generated since the new particles $\phi$ and $\chi_{i}$ do not couple to leptons. We are hence left with potential contributions to the $Z$ and photon penguins. An explicit calculation shows that the $Z$ penguin contribution vanishes. This can be explained by the chiral structure of our model with the new particles coupling only to right-handed quarks, and is also confirmed by adapting the SUSY results of [48] to our scenario. The photon penguin contribution is non-zero, however numerically small, as known from supersymmetric models [48, 49].

In summary we are left with completely SM-like rare decays like $B_{s, d} \rightarrow \mu^{+} \mu^{-}, B \rightarrow$ $K^{*} \mu^{+} \mu^{-}$and $B \rightarrow X_{s} \gamma$. Consequently the mDMFV model does not ameliorate the tension in the $B \rightarrow K^{*} \mu^{+} \mu^{-}$data.

A bit more care is however required in the case of semileptonic decays with neutrinos in the final state, such as $K \rightarrow \pi \nu \bar{\nu}$ or $B \rightarrow K^{(*)} \nu \bar{\nu}$. Since the neutrinos escape detection, the experimental signatures are $K \rightarrow \pi+\mathbb{E}_{T}$ and $B \rightarrow K^{(*)}+\mathbb{E}_{T}$ respectively. Consequently also the decays ${ }^{2} K \rightarrow \pi \chi_{\mathrm{DM}} \bar{\chi}_{\mathrm{DM}}$ and $B \rightarrow K^{(*)} \chi_{\mathrm{DM}} \bar{\chi}_{\mathrm{DM}}$, mediated by a tree level $\phi$

\footnotetext{
${ }^{2}$ We denote by $\chi_{\mathrm{DM}}$ the lightest flavor which is stable and provides the DM.
} 
exchange, will contribute to the measured branching ratio if the decay is kinematically allowed. See [50] for a detailed discussion. In order to avoid these potentially stringent constraints, in the remainder of our analysis we will assume $m_{\mathrm{DM}}>10 \mathrm{GeV}$ and therefore well outside the kinematically allowed region for these decays.

\subsection{Electroweak precision tests and electric dipole moments}

Besides the flavor violating $K$ and $B$ decays discussed above, the flavor conserving electroweak precision constraints and the bounds on electric dipole moments also put strong constraints on many NP models. In this section we consider these observables within the mDMFV model.

We start by considering electroweak precision observables. Due to the residual $\mathbb{Z}_{3}$ symmetry corrections from the mDMFV model can arise only at the loop level and are therefore suppressed by a loop factor $1 /\left(16 \pi^{2}\right)$. Furthermore the mDMFV model introduces no new $\mathrm{SU}(2)_{L}$ doublets, and only $\phi$ carries hypercharge. Consequently the contributions to electroweak precision observables receive an additional suppression by $\sim g_{Y}^{2} /\left(9 m_{\phi}^{2}\right)$. Together with the loop factor and the scale $m_{\phi}$ above the electroweak scale we conclude that all new contributions to electroweak precision observables are safely small.

Similarly we also find no significant new contribution to electric dipole moments. The reasons are as follows. Due to the chiral structure of the mDMFV model with new particles coupling only to right-handed down-type quarks no EDM is generated at the one loop level. At the two loop level a Barr-Zee type diagram [51] with $\phi$ running in the loop exists however its CP-violating phase is zero because the coupling $\lambda_{H \phi}$ is real.

\section{Flavor pre-analysis of possible structures for the DM-quark coupling}

We are now prepared to study the allowed regions of parameter space from flavor observables as well as correlations between different parameters of the coupling matrix $\lambda$.

\subsection{Strategy of the numerical analysis}

In order to determine the constraints from $\Delta F=2$ observables on the mDMFV model, we use the latest New Physics Fit results of the model-independent NP fit presented by the UTfit collaboration [52]. To this end we define

$$
M_{12}^{B_{q}}=C_{B_{q}} e^{2 i \varphi_{B_{q}}} M_{12}^{B_{q}, \mathrm{SM}} \quad(q=d, s),
$$

where $M_{12}^{B_{q}}$ is the full mixing amplitude containing both SM and mDMFV contributions. Furthermore

$$
\operatorname{Re} M_{12}^{K}=C_{\Delta M_{K}} \operatorname{Re} M_{12}^{K, \mathrm{SM}}, \quad \operatorname{Im} M_{12}^{K}=C_{\varepsilon_{K}} \operatorname{Im} M_{12}^{K, \mathrm{SM}} .
$$

These six parameters are constrained by a global fit of the NP amplitude to the available tree level and $\Delta F=2$ data $[52,53]$. In order to be conservative we impose the resulting constraints at the $2 \sigma$ level, see table 2 for a summary. In the case of $\Delta M_{K}$ we allow for a $\pm 40 \%$ uncertainty in order to capture the poorly known long distance effects. For 


\begin{tabular}{|l|l|}
\hline$\left|V_{u s}\right|=0.22527$ & $C_{\Delta M_{K}}=1.10 \pm 0.44$ \\
$\left|V_{u b}\right|=3.76 \cdot 10^{-3}$ & $C_{\varepsilon_{K}}=1.05 \pm 0.32$ \\
$\left|V_{c b}\right|=4.061 \cdot 10^{-2}$ & $C_{B_{d}}=1.07 \pm 0.34$ \\
$\delta=67.8^{\circ}$ & $\varphi_{B_{d}}=-(2.0 \pm 6.4)^{\circ}$ \\
& $C_{B_{s}}=1.066 \pm 0.166$ \\
& $\varphi_{B_{s}}=(0.6 \pm 4.0)^{\circ}$ \\
\hline
\end{tabular}

Table 2. Summary of CKM parameters and $\Delta F=2$ constraints used in our numerical analysis, see [52] for details.

consistency we set the CKM parameters to their central values obtained in the UTfit fit. All other input parameters are set to their central values listed in table 3 of [54].

Altogether, we have the following new parameters relevant for flavor violating decays:

$$
m_{\phi}, \quad m_{\chi}, \quad \lambda_{0}, \quad \lambda_{1}, \quad \lambda_{2}, \quad \theta_{12}^{\lambda}, \quad \theta_{13}^{\lambda}, \quad \theta_{23}^{\lambda}, \quad \delta_{12}^{\lambda}, \quad \delta_{13}^{\lambda}, \quad \delta_{23}^{\lambda} .
$$

Our goal is to obtain a clear picture of patterns in the coupling matrix $\lambda$ that are implied by the available $\Delta F=2$ data. To this end we fix the flavor conserving parameters $m_{\phi}, m_{\chi}$ and $\lambda_{0}$ to the values

$$
m_{\phi}=850 \mathrm{GeV}, \quad m_{\chi}=200 \mathrm{GeV}, \quad \lambda_{0}=1 .
$$

The impact of varying these parameters can be estimated from the functional dependence of $M_{12}^{i, \text { new }}\left(i=K, B_{d}, B_{s}\right)$, which is roughly given by

$$
M_{12}^{i, \text { new }} \propto \frac{\lambda_{0}^{2}}{m_{\phi}^{2}} F(x), \quad x=m_{\chi}^{2} / m_{\phi}^{2} .
$$

Note that $F(x)$ is a monotonically decreasing function varying from 1 to $1 / 3$ over the range $0<x<1$.

The $\Delta F=2$ constraints then translate directly into constraints on the values of $\xi_{K}$, $\xi_{B_{d}}$ and $\xi_{B_{s}}$, as shown in figure 3. We observe that the strongest constraints come from $K^{0}-\bar{K}^{0}$ mixing, and in particular the CP-violating parameter $\varepsilon_{K}$, which forces the phase of $\xi_{K}$ to be very close to $0, \pi / 2, \pi, 3 \pi / 2$ unless $\left|\xi_{K}\right| \lesssim 10^{-3}$. The $B$ physics constraints are less stringent and in particular do not yield a specific pattern for the phases of $\xi_{B_{q}}$. The weakest constraints are found in the $B_{s}$ system.

This pattern of allowed deviations from the SM is not specific to the mDMFV model, but can be found in all models with a generic NP flavor structure that do not induce the chirally enhanced left-right operators, like the Littlest Higgs model with T-parity analyzed in detail in [55-57]. It is a direct consequence of the CKM hierarchies that determine the size of effects within the SM, as well as the theoretical uncertainties involved. Note that e. g. in Randall-Sundrum (RS) models with bulk fermions [58-60] and left-right models [43, 61], the strong enhancement of the left-right operators in the kaon system makes the $K^{0}-\bar{K}^{0}$ constraints even more severe. 


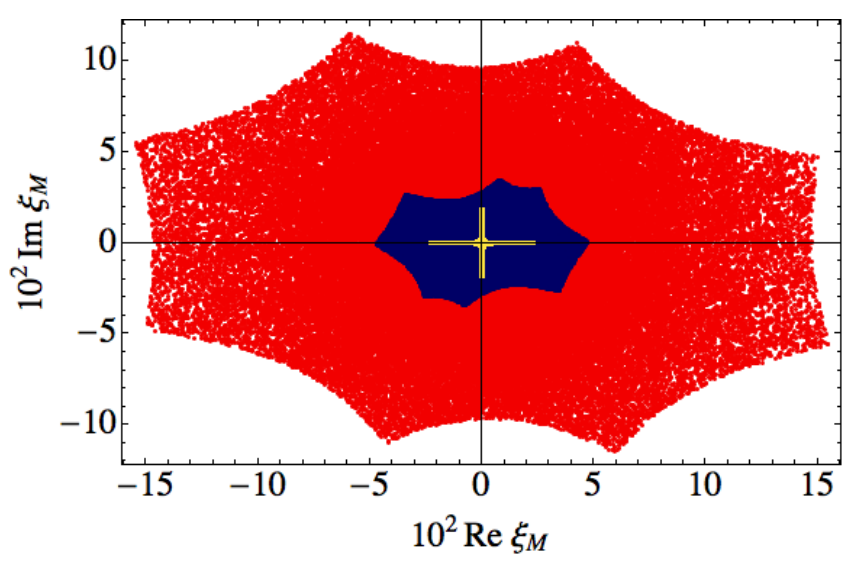

Figure 3. Allowed ranges for the flavor violating parameters $\xi_{M}=\xi_{K}$ (yellow), $\xi_{M}=\xi_{B_{d}}$ (blue), $\xi_{M}=\xi_{B_{s}}(\mathrm{red})$.

\section{2 "Flavor-safe" scenarios for the structure of $\lambda$}

We now analyze the structure of the coupling matrix $\lambda$ that is implied by the $\Delta F=$ 2 constraints. To this end we show in figure 4 the allowed points in the $\left(\lambda_{1}, \lambda_{2}, s_{12}^{\lambda}\right)$, $\left(\lambda_{1}, \lambda_{2}, s_{13}^{\lambda}\right)$ and $\left(\lambda_{1}, \lambda_{2}, s_{23}^{\lambda}\right)$ spaces, respectively.

We observe that the allowed points fall into five distinct scenarios for the structure of $\lambda$, which we discuss in some detail in the following. In order to analytically understand the scenarios, we recall the parametrization of $\lambda$ in terms of three two-flavor rotation matrices $U_{i j}$ and a diagonal matrix $D_{\lambda}$ :

$$
\lambda=U_{23} U_{13} U_{12} D_{\lambda} .
$$

1. universality scenario (black): $\lambda_{1} \simeq \lambda_{2} \simeq 0$

In this case $\lambda \simeq U_{\lambda} \cdot \lambda_{0}$ so that $\lambda \lambda^{\dagger} \simeq \lambda_{0}^{2} \cdot \mathbb{1}$. Since flavour violating effects are governed by the off-diagonal elements of $\lambda \lambda^{\dagger}$, the $\Delta F=2$ constraints are trivially fulfilled for arbitrary $U_{\lambda}$ and there are no FCNC effects beyond the SM.

2. 12-degeneracy (blue): $\lambda_{1} \simeq \lambda_{2}$

If the first two generations of DM fermions are quasi-degenerate, then - as seen from the blue points in figure 4 - the mixing angle $s_{12}^{\lambda}$ can be generic while $s_{13,23}^{\lambda}$ have to be small. This can be understood by taking the limit $\lambda_{1}=\lambda_{2}$, in which the mixing matrix $U_{12}$ becomes non-physical, and we are left with

$$
\lambda=U_{23} U_{13} D_{\lambda} .
$$

It is easy to see that in order to fully suppress flavor violating effects we need $U_{13,23} \simeq$ $\mathbb{1}$ and therefore $s_{13,23}^{\lambda} \simeq 0$.

3. 13-degeneracy (red): $\lambda_{2} \simeq-2 \lambda_{1}$

In the case $\lambda_{2} \simeq-2 \lambda_{1}$, shown by the red points in figure 4 , the first and third DM flavor are quasi-degenerate, and consequently $s_{13}^{\lambda}$ is unconstrained. In order to suppress the remaining flavor violating effects both $s_{12}^{\lambda}$ and $s_{23}^{\lambda}$ have to be small. 

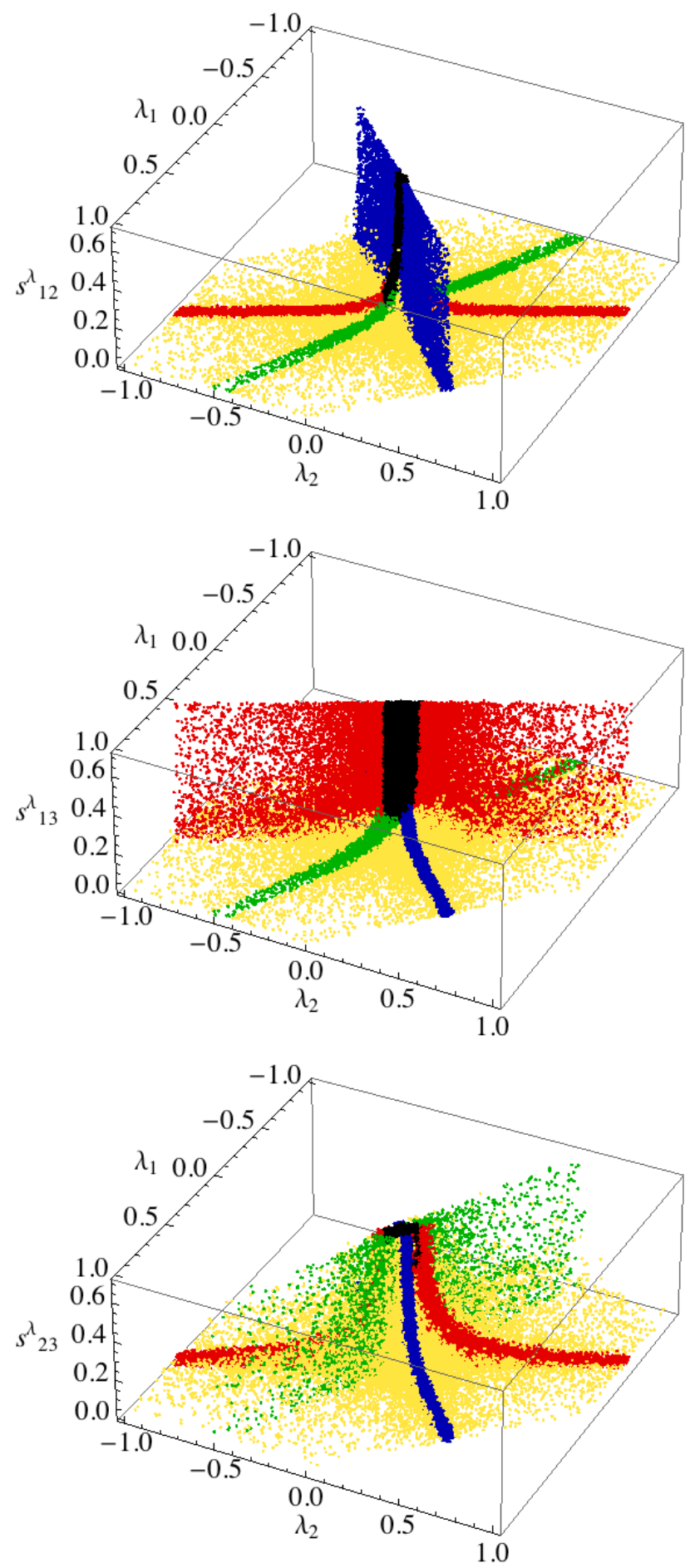

Figure 4. Scenarios for the structure of $\lambda$ : universality (black), 12-degeneracy (blue), 13degeneracy (red), 23-degeneracy (green), small mixing (yellow). 


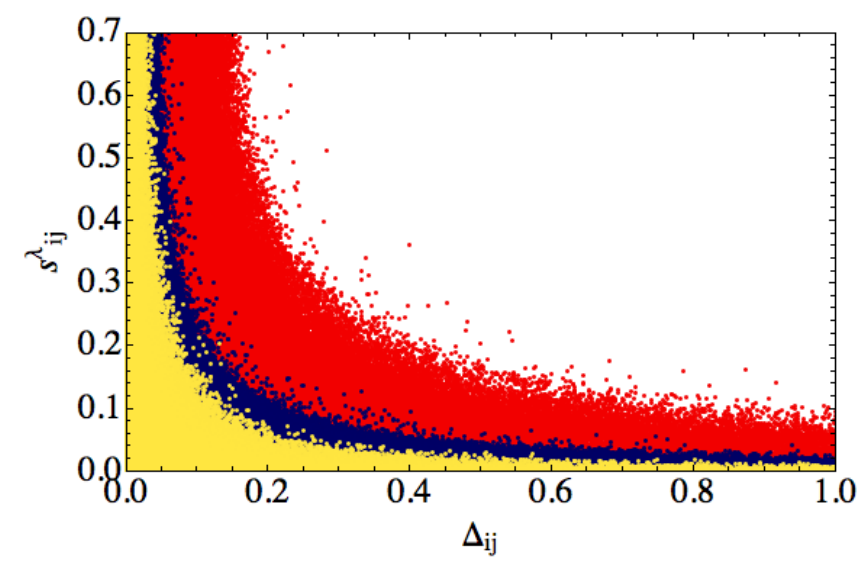

Figure 5. Allowed ranges for the mixing angles $s_{i j}^{\lambda}$ as a function of the deviation from the $i j$ degeneracy line $\Delta_{i j}=\left|D_{\lambda, i i}-D_{\lambda, j j}\right| . i j=12$ in yellow, $i j=13$ in blue, $i j=23$ in red.

4. 23-degeneracy (green): $\lambda_{2} \simeq-1 / 2 \lambda_{1}$

Finally if $\lambda_{2} \simeq-1 / 2 \lambda_{1}$, the second and third DM flavor are quasi degenerate. Consequently the mixing angle $s_{23}^{\lambda}$ is arbitrary, while $s_{12}^{\lambda}$ and $s_{13}^{\lambda}$ have to be small. This scenario is shown by the green points in figure 4 .

5. small mixing scenario (yellow): arbitrary $D_{\lambda}$

Finally if $D_{\lambda}$ does not exhibit any degeneracies, then FCNC effects have to be suppressed by the smallness of all three mixing angles $s_{12}^{\lambda} \simeq s_{13}^{\lambda} \simeq s_{23}^{\lambda} \simeq 0$. This scenario, shown by the yellow points in figure 4 , corresponds to a diagonal but non-degenerate coupling matrix $\lambda$.

In order to quantify the allowed size of deviations from the degeneracy scenarios discussed above, we show in figure 5 the mixing angles $s_{i j}^{\lambda}$ as a function of the deviation from the corresponding degeneracy line. We observe that the constraint on 12 and 13 mixings are comparable with the former being somewhat stronger, while the constraint on the 23 mixing angle is significantly weaker. This is a direct consequence of the allowed sizes of NP effects in the various meson systems, see figure 3.

\subsection{A note on flavor safety of the UV completion}

FCNC processes are known to be sensitive to NP at very high scales. It is therefore questionable whether a study of the simplified mDMFV model is sufficient to capture all relevant effects.

Following the DMFV principle we can write any contribution from the UV completion in terms of higher-dimensional operators that are suppressed by powers of the UV scale $\Lambda_{\mathrm{UV}}$ and made formally invariant under the flavor group (2.1) by insertion of the appropriate combination of spurion fields $Y_{u, d}$ and $\lambda$. The leading contribution to the $\Delta F=2$ effective Hamiltonian is then

$$
\mathcal{H}_{\mathrm{eff}}^{\Delta F=2, \mathrm{UV}} \sim \frac{c_{\Delta F=2}^{\mathrm{UV}}}{\Lambda_{\mathrm{UV}}^{2}} \lambda \lambda^{\dagger}\left(\bar{s} \gamma^{\mu} P_{R} d\right)\left(\bar{s} \gamma_{\mu} P_{R} d\right)
$$


where $c_{\Delta F=2}^{\mathrm{UV}}$ is an $\mathcal{O}(1)$ coefficient that is common to all three meson systems. Comparing this to the new contribution generated first at the one loop level in the simplified model (see (4.1)), that can schematically be written as

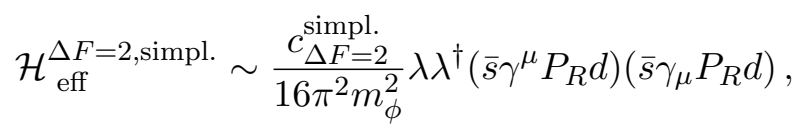

we observe that both contributions carry the same flavor structure. Furthermore the UV contribution is suppressed with respect to the simplified model one if

$$
\Lambda_{\mathrm{UV}} \gtrsim 4 \pi m_{\phi} .
$$

Therefore, NP close to the mass of $\phi$ does not change the flavor phenomenology as long as it respects the DMFV hypothesis. Generic flavor violation needs to be suppressed by a much higher scale $\sim \mathcal{O}(100-1000) \mathrm{TeV}[62]$.

\subsection{Recovering the MFV limit in the structures for $\lambda$}

Earlier studies of flavored DM have been restricted to the MFV framework in order to be safe from undesired effects in flavor observables. The flavor-safe scenarios identified above are more general than the MFV ansatz. It is also worthwhile to study how MFV can be recovered in the DMFV framework.

Let us first consider the case where $\mathrm{U}(3)_{\chi}$ is identified with $\mathrm{U}(3)_{d}$. The MFV hypothesis then requires that $\lambda$ takes the schematic form

$$
\lambda \propto \mathbb{1}+\alpha Y_{d}^{\dagger} Y_{d}+\ldots,
$$

where $\alpha$ is an arbitrary coefficient. The matrix $\lambda$ is diagonal in the down quark mass basis. In particular $Y_{d}$ is proportional to the down-type quark masses. Considering that $Y_{d}$ can be approximated by $Y_{d} \sim \operatorname{diag}\left(0,0, y_{b}\right), \mathrm{MFV}$ must be close to the 12-degeneracy. Additionally MFV requires

$$
m_{\chi} \propto \mathbb{1}+\beta Y_{d}^{\dagger} Y_{d}+\ldots,
$$

where $\beta$ is an arbitrary coefficient. The same expansion for $m_{\chi}$ is obtained when inserting (5.11) into the DMFV expansion (3.11), so that MFV in this case is consistent with the DMFV hypothesis. As $\lambda$ and $m_{\chi}$ are diagonal in the same basis, all three flavor mixing angles are zero. Thus the MFV limit can be recovered as a very specific subset of parameter space, (determined by the specific choice of $Y_{d}$ ) close to the 12-degeneracy line with all mixing angles zero.

If instead $\mathrm{U}(3)_{\chi}$ is identified with $\mathrm{U}(3)_{u}$, then

$$
\lambda \propto Y_{d}^{\dagger} Y_{u}+\ldots,
$$

while

$$
m_{\chi} \propto \mathbb{1}+\alpha Y_{u}^{\dagger} Y_{u}+\ldots,
$$

where as before $\alpha$ is an arbitrary coefficient. We can see immediately that the mass splittings are not directly correlated with the $\lambda$ matrix, as was the case for DMFV (3.11) with the separate $\mathrm{U}(3)_{\chi}$ symmetry. 
Finally identifying $\mathrm{U}(3)_{\chi}$ with $\mathrm{U}(3)_{q}$, we have

$$
\lambda \propto Y_{d}+\ldots
$$

and

$$
m_{\chi} \propto \mathbb{1}+\alpha Y_{u} Y_{u}^{\dagger}+\beta Y_{d} Y_{d}^{\dagger}+\ldots,
$$

where $\alpha$ and $\beta$ are arbitrary coefficients. Again we observe that the pattern of splittings in $m_{\chi}$ are not directly correlated with the $\lambda$ matrix.

In summary we find that if $\chi$ is assumed to transform under $\mathrm{U}(3)_{d}$ then the MFV limit can be recovered as a small subset of the scenarios for $\lambda$, with an approximate 12degeneracy and all mixing angles identically zero. On the other hand, the MFV limits for $\chi$ transforming under $\mathrm{U}(3)_{u}$ or $\mathrm{U}(3)_{q}$ have mass splitting patterns which are not correlated with the coupling matrix $\lambda$, and therefore to capture these cases one needs to consider additional contributions to the mass splitting in (3.11).

\section{From the flavor pre-analysis to dark matter scenarios}

Our flavor pre-analysis shows that a generic coupling matrix $\lambda$ leads to unacceptably large corrections to $\Delta F=2$ observables. We have identified a number of non-trivial scenarios for the structure of $\lambda$ for which flavor violating effects are efficiently suppressed:

1. Universality scenario: all elements of the diagonal matrix $D_{\lambda}$ equal and arbitrary flavor mixing angles.

2. $i j$-degeneracy scenarios $(i j=12,13,23): D_{\lambda, i i}=D_{\lambda, j j}$, arbitrary $s_{i j}^{\lambda}$ and the other mixing angles small.

3. Small mixing scenario: small mixing angles and arbitrary $D_{\lambda}$.

While these scenarios have been identified in a scan with fixed flavor conserving parameters $m_{\phi}, m_{\chi}$ and $\lambda_{0}$, we stress that these structures for $\lambda$ also remain valid for different choices of parameters. Furthermore, even though our analysis has been performed within the simplified framework of the mDMFV model, the identified scenarios for $\lambda$ remain flavor-safe in non-minimal versions of DMFV also. Thus they provide a useful framework for future study of the phenomenology of DMFV models - employing any of these scenarios for the structure of $\lambda$ efficiently evades all FCNC constraints, without the need for an involved study of the latter.

In table 3 we summarize the flavor-safe scenarios for $\lambda$ and their implications for the mass pattern in the DM sector. It is clear that flavor constraints do not impose a specific mass hierarchy on the dark sector, i. e. from the point of FCNC constraints any dark flavor can be the lightest. Note that an exact degeneracy of two flavors is unnatural, since in the case of universal $\lambda$ it is violated by the presence of $Y_{d, u}$ at higher orders in the DMFV expansion. We therefore assume that the observed DM is composed of a single $\chi$ flavor, while the decay of the heavier states is fast enough to have happened in the early universe. We refer the reader to appendix D for an estimate of the life-time of the heavier states. 


\begin{tabular}{|l|l|l|}
\hline scenario & specification & lightest DM particle \\
\hline universal scenario $\left(m_{\chi_{d}} \simeq m_{\chi_{s}} \simeq m_{\chi_{b}}\right)$ & - & all hierarchies possible \\
\hline 12 degeneracy $\left(m_{\chi_{d}} \simeq m_{\chi_{s}}\right)$ & $\eta \lambda_{1}>0$ & $\chi_{b}$ \\
& $\eta \lambda_{1}<0$ & $\chi_{d}$ or $\chi_{s}$ \\
\hline 13 degeneracy $\left(m_{\chi_{d}} \simeq m_{\chi_{b}}\right)$ & $\eta \lambda_{1}>0$ & $\chi_{s}$ \\
& $\eta \lambda_{1}<0$ & $\chi_{d}$ or $\chi_{b}$ \\
\hline 23 degeneracy $\left(m_{\chi_{s}} \simeq m_{\chi_{b}}\right)$ & $\eta \lambda_{1}>0$ & $\chi_{s}$ or $\chi_{b}$ \\
& $\eta \lambda_{1}<0$ & $\chi_{d}$ \\
\hline small mixing scenario & - & all hierarchies possible \\
\hline
\end{tabular}

Table 3. Overview of flavor-safe scenarios and their implications for the mass hierarchy in the DM sector.

However not all DM flavors are equally motivated from the point of DM and collider phenomenology. DM that couples dominantly to first generation quarks, like $d$-flavored DM, is strongly constrained by the direct detection experiments. If the DM relic density is assumed to arise from thermal freeze-out in the early universe, the relic abundance condition is in severe tension with the experimental constraints. We will therefore not consider the case of $d$-flavored DM further.

As far as direct detection constraints are concerned, $s$ - and $b$-flavored DM are on equal footing. Interestingly the same holds, at least qualitatively, also for the flavor phenomenology - as we have seen in figure 5 the amount of flavor violation allowed by $\Delta F=2$ constraints is almost symmetric under the exchange of the second and third generation, $2 \leftrightarrow 3$.

The case is however different for collider phenomenology. While pair production of the mediator and its subsequent decay will dominantly produce light jets and missing energy in the $s$-flavored case, in the case of $b$-flavored DM the large coupling to the $b$ quark will give rise to $b$-jet signatures in a significant fraction of the events. Since events with $b$-jets are much more easily distinguished from the QCD background, the collider phenomenology of $b$-flavored DM is at the same time more constraining (in particular concerning the bound on the mediator mass) and also more promising, as quite distinctive signatures arise.

A further motivation for $b$-flavored DM comes from indirect detection. Recently it has been shown that a $35 \mathrm{GeV} \chi_{b}$ provides a good fit to the excess $\gamma$-rays observed at the galactic center [21].

Therefore, in the rest of our analysis we restrict ourselves to the case of $b$-flavored DM, i.e. $m_{\chi_{b}}<m_{\chi_{d, s}}$. We also assume that the DM relic abundance is set by the thermal freeze-out condition, so that $D_{\lambda, 33}$ has to be large. Due to the strong constraints on the first generation coupling from direct detection and collider data, we deduce that $D_{\lambda, 11}<D_{\lambda, 33}$. Consequently in order to ensure the correct mass hierarchy, we have $\eta<0$.

We are then left with the following scenarios for DM freeze-out:

1. single flavor freeze-out: the $s$ - and $d$-flavored states are split from the $b$-flavored DM by at least $10 \%$. 


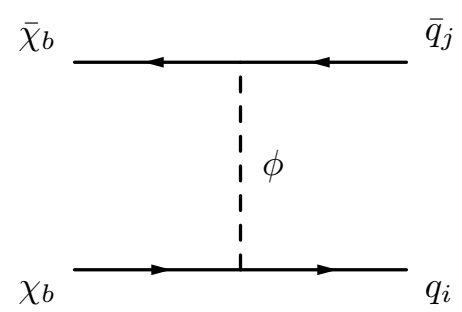

Figure 6. Feynman diagram for dark matter annihilation in the early universe.

2. two flavor freeze-out:

(a) 13-degeneracy - $\chi_{b}$ and $\chi_{d}$ are quasi-degenerate, while $\chi_{s}$ is split

(b) 23-degeneracy $-\chi_{b}$ and $\chi_{s}$ are quasi-degenerate, while $\chi_{d}$ is split

3. three flavor freeze-out: all three states are quasi-degenerate. Such a scenario can either be achieved by a quasi universal coupling matrix $D_{\lambda}$, or if the DMFV expansion parameter $\eta$ is loop-suppressed, $|\eta| \sim 10^{-2}$.

In our numerical analysis we will study all of these scenarios in turn.

\section{$7 \quad$ Phenomenology of $b$-flavored dark matter}

In this section, we study the constraints arising from requiring the DM to be a thermal relic and from direct detection experiments. We note that the relic abundance constraints may be potentially relaxed in the presence of other particles in the dark sector.

The presence of multiple flavors can affect the DM freeze-out significantly. This occurs when the mass splitting between different flavors of DM is much smaller than the freeze-out temperature, $\left(T_{f} \sim m_{\chi} / 20\right)$. For mass splittings much bigger than this scale, the freeze-out follows the standard WIMP paradigm.

We show that in the range of parameter space we consider, the heavier DM flavors decay before big bang nucleosynthesis (BBN) (see appendix D). The direct detection constraints then depend sensitively on the couplings of the lightest flavor of DM. In particular, when the lightest dark flavor couples appreciably to the first generation quarks, it gives rise to a very large direct detection signal. If this contribution is suppressed, the dominant contribution then arises at 1-loop level, which is seen to be within the reach of present and future direct detection experiments.

\subsection{Relic abundance}

We will consider two different qualitative regimes. When their masses are nearly degenerate, then all DM flavors are present during freeze-out and can be treated together. Otherwise only the lightest flavor of DM remains in the thermal bath. 
We start with a single flavor freeze-out. The dominant annihilation during freeze-out occurs in the lowest partial wave. In this limit

$$
\langle\sigma v\rangle_{b b}=\sum_{i, j} \frac{3 \lambda_{i b} \lambda_{i b}^{*} \lambda_{j b} \lambda_{j b}^{*} m_{\chi_{b}}^{2}}{32 \pi\left(m_{\chi_{b}}^{2}+m_{\phi}^{2}\right)^{2}}=\frac{3\left(D_{\lambda}^{\dagger} U^{\dagger} U D_{\lambda}\right)_{33}^{2} m_{\chi_{b}}^{2}}{32 \pi\left(m_{\chi_{b}}^{2}+m_{\phi}^{2}\right)^{2}}=\frac{3 D_{33}^{4} m_{\chi_{b}}^{2}}{32 \pi\left(m_{\chi_{b}}^{2}+m_{\phi}^{2}\right)^{2}}
$$

where we have ignored the masses of the final state quarks.

The relic abundance is determined by solving the Boltzmann equation for the DM number density $n$ at late times. For a Dirac fermion, it is useful to convert the annihilation cross section into an effective cross section [63, 64].

$$
\langle\sigma v\rangle_{\mathrm{eff}}=\frac{1}{2}\langle\sigma v\rangle .
$$

which is approximately required to be [65]

$$
\langle\sigma v\rangle_{\mathrm{eff}}=2.2 \times 10^{-26} \mathrm{~cm}^{3} / \mathrm{s}
$$

in order to produce the correct relic abundance of DM.

Next we consider the case, where the mass splitting between the DM flavors is much smaller than the temperature at freeze-out. Consequently, we have to take into account the co-annihilation between different flavors. We assume that flavor changing (but DM number preserving) interactions $\chi_{i} q \rightarrow \chi_{j} q$ are fast during the epoch of DM freeze-out. The rate for these processes is enhanced over the DM annihilations - which are approximately in thermal equilibrium - by a large Boltzmann factor $\left(\mathcal{O}\left(10^{9}\right)\right)$. Thus, this approximation is valid as long as any individual cross sections are not suppressed enough to overwhelm this factor.

Then the Boltzmann equation for freeze-out has a very similar form to the single DM case, and can be solved in exactly the same way. The relic abundance is in fact relatively insensitive to the change in the number of DM species, changing by only about $5 \%$ when other parameters are kept fixed. In the limit of small splitting, the effective cross section is well approximated by [63],

$$
\langle\sigma v\rangle_{\mathrm{eff}}=\frac{1}{18} \sum_{i, j=d, s, b}\langle\sigma v\rangle_{i j}
$$

The co-annihilation cross section can be derived by modifying equation (7.1), e.g.

$$
\langle\sigma v\rangle_{b s}=\sum_{i, j} \frac{3 \lambda_{i s} \lambda_{i s}^{*} \lambda_{j b} \lambda_{j b}^{*} m_{\chi b}^{2}}{32 \pi\left(m_{\chi b}^{2}+m_{\phi}^{2}\right)^{2}}=\frac{3 D_{22}^{2} D_{33}^{2} m_{\chi b}^{2}}{32 \pi\left(m_{\chi b}^{2}+m_{\phi}^{2}\right)^{2}} .
$$

Note that the splitting between DM masses is in this case negligible $\left(m_{\chi d} \simeq m_{\chi_{s}} \simeq m_{\chi b}\right)$.

If only two states are nearly degenerate, then a two flavor freeze-out occurs. The corresponding formulae can be straightforwardly obtained from the above results. 

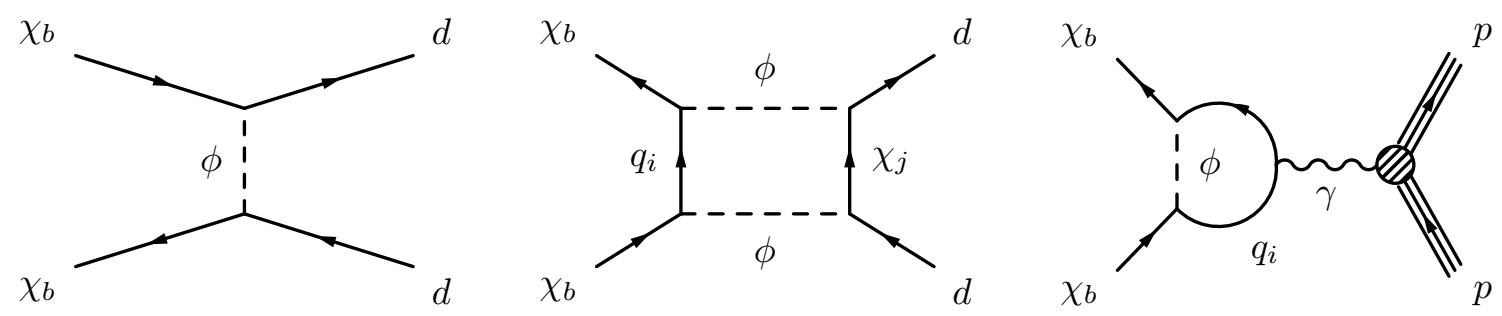

Figure 7. Diagrams contributing to WIMP-nucleon scattering in the mDMFV model.

\subsection{Direct detection}

For $b$-flavored DM, the direct detection scattering arises either through mixing, or at one loop.

We focus on the spin-independent contribution to the WIMP-nucleus scattering. The reported experimental bounds are translated to the WIMP-nucleon cross section, which can be written as

$$
\sigma_{n}^{\mathrm{SI}}=\frac{\mu_{n}^{2}}{\pi A^{2}}\left|Z f_{p}+(A-Z) f_{n}\right|^{2}
$$

where $\mu_{n}$ is the reduced mass of the WIMP-nucleon system, $A$ and $Z$ are the mass and atomic numbers of the nucleus respectively, and $f_{n}$ and $f_{p}$ parametrize DM coupling to neutrons and protons. The relevant processes are shown in figure 7.

For direct detection, we can safely work in the effective theory with the $\phi$ integrated out. The Lorentz structure of the four-fermion operator generated after performing the Fierz transformation is given by

$$
\bar{\chi}_{b} \gamma^{\mu}\left(1-\gamma^{5}\right) \chi_{b} \bar{d} \gamma^{\mu}\left(1+\gamma^{5}\right) d .
$$

There are three contributions to $f_{n, p}$ :

$$
f_{n, p}=f_{n, p}^{\text {tree }}+f_{n, p}^{\text {box }}+f_{n, p}^{\text {photon }} .
$$

These contributions are individually given as follows:

1. $s$-channel $\phi$ at tree-level:

In presence of significant mixing in the $\lambda$ matrix, this is the dominant contribution to direct detection:

$$
2 f_{p}^{\text {tree }}=f_{n}^{\text {tree }}=\frac{\left|\lambda_{d b}\right|^{2}}{4 m_{\phi}^{2}} .
$$

The spin-independent part in equation (7.7) arises from the matrix element of the quark vector current bilinear in the nucleons. Thus, only the valence $d$-quark contributes.

2. One-loop photon exchange:

The interaction of DM with nucleons via photon exchange is conveniently parametrized as the electromagnetic form factors of the DM coupling with those of the 
nucleus. In particular, the scattering cross sections arise from charge-charge, dipolecharge and dipole-dipole interactions [4]. In the region of interest, the charge-charge interactions dominate, leading to

$$
f_{p}^{\text {photon }}=\sum_{i} \frac{\left|\lambda_{i b}\right|^{2} e^{2}}{96 \pi^{2} m_{\phi}^{2}} \log \left[\frac{m_{q_{i}}^{2}}{m_{\phi}^{2}}\right]
$$

in the leading-log approximation.

3. Box diagram with $\phi$ exchange in the t-channel:

This new contribution depends upon the coupling of DM with the first generations quarks [8] and is given by

$$
4 f_{p}^{\text {box }}=2 f_{n}^{\text {box }}=\sum_{i, j} \frac{\left|\lambda_{d j}\right|^{2}\left|\lambda_{i b}\right|^{2}}{16 \pi^{2} m_{\phi}^{2}} F\left(\frac{m_{q_{i}}^{2}}{m_{\phi}^{2}}, \frac{m_{\chi_{j}}^{2}}{m_{\phi}^{2}}\right),
$$

with the loop function $F$ given in appendix C.

The tree-level contribution, being flavor violating, constrains the mixing $s_{13}^{\lambda}$ to be small. The LUX experiment [39] is sensitive to even the loop level scattering cross sections for WIMP DM. These contributions are present even in the absence of flavor violation. The box and the photon loop diagrams are seen to destructively interfere.

\section{Combined numerical analysis of flavor and dark matter constraints}

Having all relevant formulae for the DM phenomenology in hand, we are now ready to perform a combined numerical analysis of both DM and flavor constraints. We restrict ourselves to the phenomenologically most interesting case of $b$-flavored DM, and study in turn the scenarios identified in section 6 .

The DM mass $m_{\chi_{b}}$ is allowed to vary in the phenomenologically interesting region $10 \mathrm{GeV}<m_{\chi_{b}}<250 \mathrm{GeV}$. We also assume $\eta<0$ in order to suppress the $\chi$ coupling to the first generation, in order to cope with the strong direct detection and collider constraints. Convergence of the DMFV expansion is ensured by requiring $\left|\eta D_{\lambda, 33}^{2}\right|<0.3$. Finally, since corrections to $m_{\chi}$ are unavoidably generated at the one loop level, we take $|\eta|>10^{-2}$. In summary,

$$
-\frac{0.3}{D_{\lambda, 33}^{2}}<\eta<-0.01
$$

We fix $m_{\phi}=850 \mathrm{GeV}$ in agreement with the collider constraints, see section 9 . The parameters of the coupling matrix $\lambda$ are scattered, imposing the flavor and DM constraints from section 4 and 7 .

\subsection{Single flavor freeze-out}

If the masses of the heavier flavors $\chi_{d, s}$ are sufficiently split from the DM mass $m_{\chi_{b}}$ (by $\gtrsim 10 \%$ ), then at the freeze-out temperature only the lightest state $\chi_{b}$ is left while the heavier ones have decayed. Therefore the single flavor freeze-out condition for the relic 

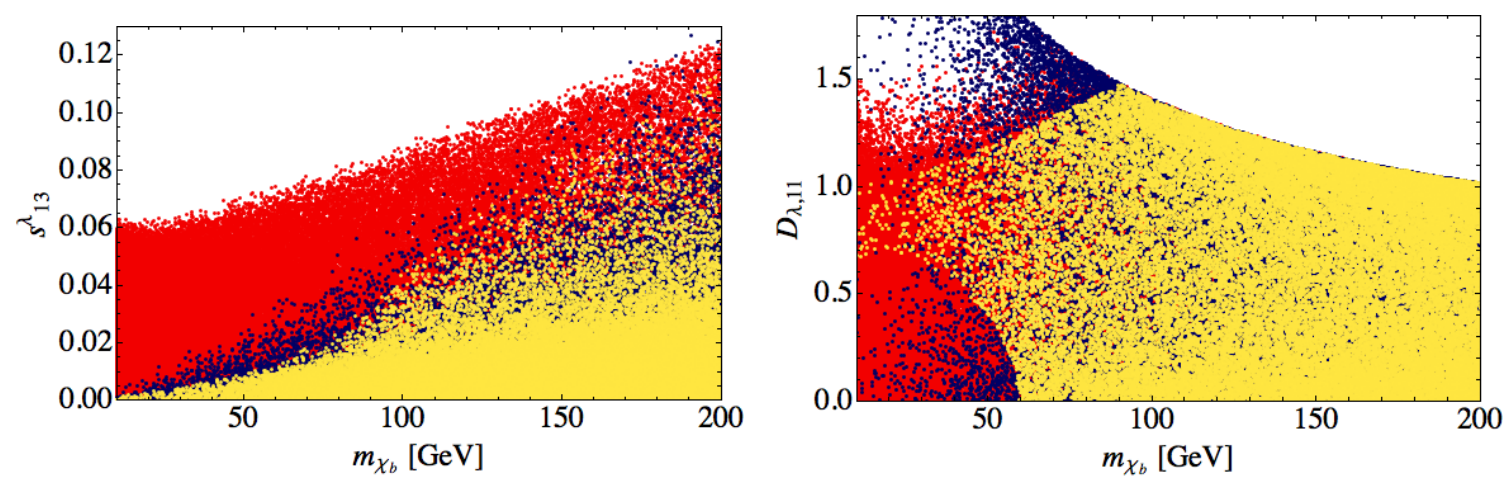

Figure 8. Flavor mixing angle $s_{13}^{\lambda}$ and first generation coupling $D_{\lambda, 11}$ as functions of the DM mass $m_{\chi_{b}}$ in the single flavor freeze-out scenario. The mass hierarchy $m_{\chi_{b}}<m_{\chi_{d, s}}$ and the relic abundance constraint are imposed. The red points satisfy the bound from LUX, while the blue points satisfy the $\Delta F=2$ constraints. For the yellow points both LUX and $\Delta F=2$ constraints are imposed.

abundance, eq. (7.3), applies and fixes $D_{\lambda, 33}$ as a function of $m_{\chi_{b}}$. The couplings $D_{\lambda, 11}$ and $D_{\lambda, 22}$ on the other hand are free to vary. They both need to be split from $D_{\lambda, 33}$ in order to achieve the mass splitting.

Figure 8 shows the result of a parameter scan over $m_{\chi_{b}}$ and $\lambda$, with the relic abundance constraint for the single flavor freeze-out scenario imposed. The red points satisfy the bound from LUX, while the blue points satisfy the $\Delta F=2$ constraints. The yellow points fulfill both LUX and $\Delta F=2$ constraints.

From the left panel, showing $s_{13}^{\lambda}$ as a function of $m_{\chi_{b}}$, we can see that both the LUX and the $\Delta F=2$ constraints require $s_{13}^{\lambda}$ to be small. In case of LUX this constraint arises from the necessary suppression of the DM-nucleon scattering at tree level. The $\Delta F=2$ constraints on the other hand require $s_{13}^{\lambda}$ to be small, as the mass splitting between $\chi_{d}$ and $\chi_{b}$ requires a deviation from the 13-degeneracy scenario $D_{\lambda, 11}=D_{\lambda, 33}$. This bound becomes stronger for small $m_{\chi_{b}}$ for the following reason: small $m_{\chi_{b}}$ requires a large $D_{\lambda, 33}$ from the relic abundance constraint, so that $|\eta|_{\text {max }}$ decreases with decreasing $m_{\chi_{b}}$. In turn a larger splitting between $D_{\lambda, 11}$ and $D_{\lambda, 33}$ is required in order to generate the $\gtrsim 10 \%$ mass splitting, implying smaller flavor mixing $s_{13}^{\lambda}$. We further observe that once both the LUX and the $\Delta F=2$ constraints are taken into account, the upper bound on $s_{13}^{\lambda}$ gets stronger than the individual ones. This proves a non-trivial interplay of the flavor and DM constraints and underlines the importance of a combined study.

In the right panel we show the allowed size of $D_{\lambda, 11}$ as a function of $m_{\chi_{b}}$. The upper bound on $D_{\lambda, 11}$ as a function of $m_{\chi_{b}}$ arises from the relic abundance constraint on $D_{\lambda, 33}$ together with the hierarchy requirement $D_{\lambda, 11}<D_{\lambda, 33}$. For $m_{\chi_{b}} \gtrsim 100 \mathrm{GeV}$ the whole range of $D_{\lambda, 11}$ up to the relic abundance bound is allowed. However for smaller $m_{\chi_{b}}$ the LUX constraint disfavors large values for $D_{\lambda, 11}$, so that in combination with the $\Delta F=2$ constraints a sharp cutoff arises. This cutoff decreases with decreasing mass $m_{\chi_{b}}$. Below $m_{\chi_{b}} \sim 60 \mathrm{GeV}$ also a lower bound on $D_{\lambda, 11}$ arises for the parameter points that satisfy both the LUX and flavor constraints - interestingly this bound does not emerge if the 
constraints are taken into account separately. This is another clear sign of a non-trivial interplay of the flavor and DM constraints.

Analytically the bounds on $D_{\lambda, 11}$ can be understood by having a closer look at the structure of the WIMP-nucleon cross-section in the mDMFV model. As already mentioned above, the $s$-channel $\phi$ exchange at tree level is proportional to $\left(D_{\lambda, 33} s_{13}^{\lambda}\right)^{2}$ and therefore places a strong constraint on $s_{13}^{\lambda}$. We are then left with the one-loop box and photon penguin contributions. The box amplitude is positive and proportional to

$$
D_{\lambda, 33}^{2} \cdot\left[\left(D_{\lambda, 11} c_{12}^{\lambda}\right)^{2}+\left(D_{\lambda, 22} s_{12}^{\lambda}\right)^{2}\right]
$$

It can therefore be suppressed by choosing both terms in the bracket small. This explains why the LUX constraint, which is strongest for low DM masses, gives rise to an upper bound on $D_{\lambda, 11}$.

The size of the photon penguin is determined by $D_{\lambda, 33}^{2}$ and therefore fixed by the relic abundance (there is a small dependence on $s_{23}^{\lambda}$, due to the difference in quark masses). Interestingly due to the log factor the photon penguin amplitude carries an overall minus sign. This relative sign between the penguin and box amplitudes leads to a cancellation of the two contributions, provided the expression in the bracket of (8.2) is of the right size. In the absence of flavor constraints $D_{\lambda, 11}, D_{\lambda, 22}$ and $s_{12}^{\lambda}$ are independent parameters. Consequently no conclusion can be drawn on the value of only one of them, while letting the other two vary. Taking into account the flavor constraints the picture changes however. In that case $s_{12}^{\lambda}$ is allowed to be sizable only if $D_{\lambda, 22} \simeq D_{\lambda, 11}$. Eq. (8.2) then reduces to $D_{\lambda, 33}^{2} \cdot D_{\lambda, 11}^{2}$, and we can directly read off the requirement that $D_{\lambda, 11}$ has to lie in a specific range such that the cancellation between the penguin and box contributions can work.

This mechanism provides a realization of xenophobic DM [40,41]. The box diagram contributes to DM scattering off protons and neutrons, while the photon penguin is only present for DM-proton scattering. Therefore the penguin-box cancellation works only for a specific number of protons and neutrons in the nucleus.

We note that a pattern of constraints analogous to the one observed in figure 8 arises for all the possible mass hierarchies in the dark sector, i. e. it does not depend qualitatively on whether a single flavor or multiple flavors are present at freeze-out.

Next let us recover which of the flavor scenarios identified in section 5 are realized in the single flavor freeze-out case. To this end we parameterize the matrix $D_{\lambda}$ in terms of the parameters $\lambda_{0,1,2}$, used in the flavor analysis, and defined in (3.6). The distribution of parameter points consistent with the LUX and flavor constraints in the $\lambda_{1}-\lambda_{2}$-plane is shown in figure 9 . We observe that the requirement $m_{\chi_{b}}<m_{\chi_{d, s}}$ and therefore $D_{\lambda, 11}, D_{\lambda, 22}<$ $D_{\lambda, 33}$ restricts the allowed parameter space to the region $\lambda_{2}<-1 / 2 \lambda_{1} \wedge \lambda_{2}<-2 \lambda_{1}$. Consequently only the 12 degeneracy scenario and the small mixing scenario are realized in this case. The DM mass $m_{\chi_{b}}$ is indicated by the color, with the dark points corresponding to light DM. We see that with smaller DM mass $m_{\chi_{b}}$ points move further away from the universality $\lambda_{1}=\lambda_{2}=0$. This feature is a direct consequence of the upper bound on $D_{\lambda, 11}$ for $m_{\chi_{b}} \lesssim 100 \mathrm{GeV}$, requiring a sizable splitting in $D_{\lambda}$. 


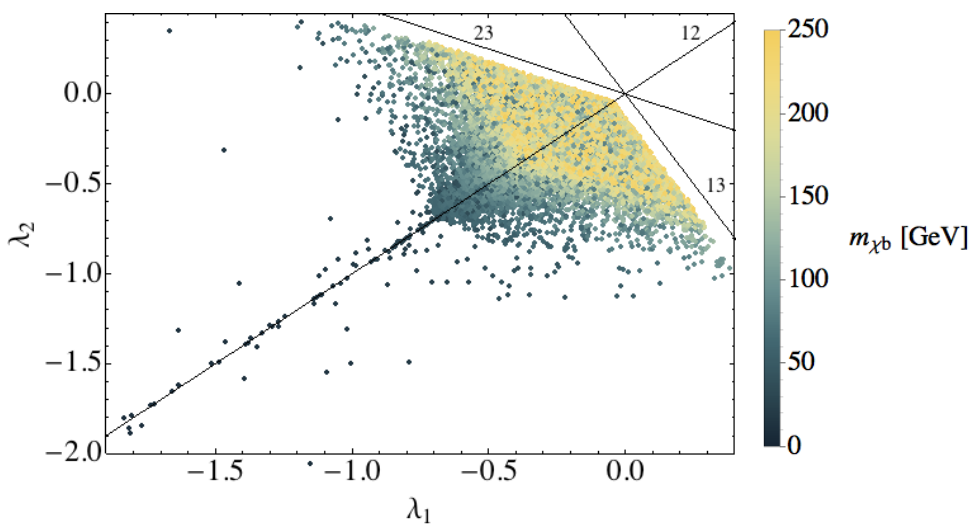

Figure 9. Allowed region in the $\lambda_{1}-\lambda_{2}$-plane for the single flavor freeze-out scenario, after imposing the relic abundance, LUX and flavor constraints. The DM mass $m_{\chi_{b}}$ is indicated by the color, and the $i j$-degeneracy lines are sketched.

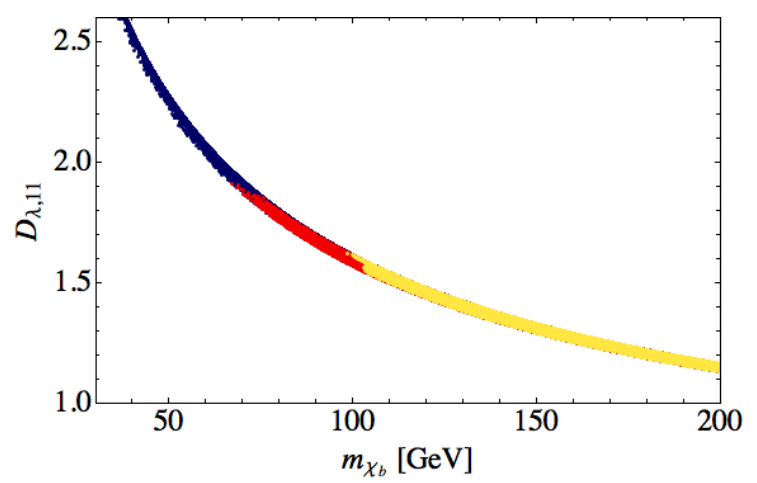

Figure 10. First generation coupling $D_{\lambda, 11}$ as function of the DM mass $m_{\chi_{b}}$ in the 13-degeneracy scenario. The mass hierarchy $m_{\chi_{b}} \lesssim m_{\chi_{d}}<m_{\chi_{s}}$ and the relic abundance constraint are imposed. The red points satisfy the bound from LUX, while the blue points satisfy the $\Delta F=2$ constraints. For the yellow points both LUX and $\Delta F=2$ constraints are imposed.

\subsection{3-degeneracy}

Next we consider the scenario of quasi-degenerate first and third dark generations, i.e. $m_{\chi_{b}} \lesssim m_{\chi_{d}}<m_{\chi_{s}}$. In this case we have $D_{\lambda, 33} \gtrsim D_{\lambda, 11}>D_{\lambda, 22}$. Comparing this structure to the scenarios identified in the flavor pre-analysis we see that we are confined to the 13-degeneracy scenario, in which $s_{12}^{\lambda}$ and $s_{23}^{\lambda}$ are small while $s_{13}^{\lambda}$ is in principle allowed to be large. However recalling the results from the previous section we anticipate that once the LUX bound is imposed also the latter mixing angle is constrained to be small, in order to suppress the tree level contribution to the WIMP-nucleon scattering. This is indeed confirmed by our numerical analysis.

In figure 10 we show the allowed values of the first generation coupling $D_{\lambda, 11}$ as a function of the DM mass $m_{\chi_{b}}$. We observe that due to the quasi-degeneracy of the first and third generation for a given $m_{\chi_{b}}$ only a small range of parameter space is allowed by the relic abundance constraint. Consequently the upper bound on $D_{\lambda, 11}$ that arises again from the need to cut off the box contribution to the WIMP-nucleon scattering translates 


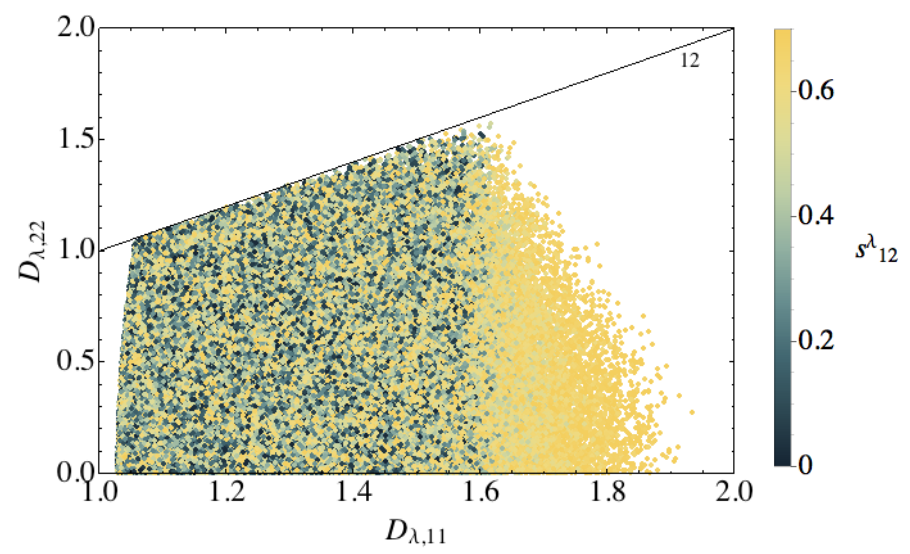

Figure 11. Allowed region for $D_{\lambda, 11}$ and $D_{\lambda, 22}$ in the 13-degeneracy scenario, after imposing both the relic abundance and LUX constraints, while the flavor constraints are not taken into account. The flavor mixing angle $s_{12}^{\lambda}$ is indicated by the color, and the 12 -degeneracy line $D_{\lambda, 11}=D_{\lambda, 22}$ is sketched.

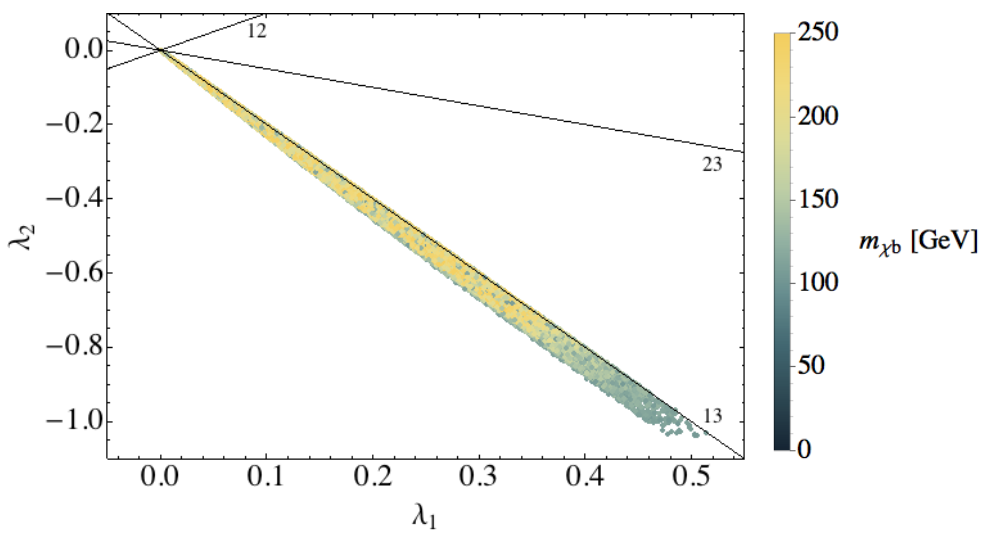

Figure 12. Allowed region in the $\lambda_{1}-\lambda_{2}$-plane for the 13-degeneracy scenario, after imposing the relic abundance, LUX and flavor constraints. The DM mass $m_{\chi_{b}}$ is indicated by the color, and the $i j$-degeneracy lines are sketched.

into a lower bound on $m_{\chi_{b}}$. If we neglect the flavor constraints and take into account only the LUX bound, we find a lower bound $m_{\chi_{b}} \gtrsim 70 \mathrm{GeV}$. Taking into account both the LUX and flavor constraints simultaneously, the bound becomes considerably stronger, $m_{\chi_{b}} \gtrsim 100 \mathrm{GeV}$. Again we stress the non-trivial interplay of flavor and DM constraints.

The interplay of flavor and DM constraints can be understood from figure 11. We show the allowed parameter range in the $D_{\lambda, 11}-D_{\lambda, 22}$-plane, applying only the LUX bound, but not the flavor constraints. We observe that the largest values for $D_{\lambda, 11}$, corresponding to the smallest DM masses, are reached away from the 12-degeneracy line and require nearmaximal mixing $s_{12}^{\lambda}$. This part of parameter space, while perfectly consistent with the DM constraints, is however ruled out by the stringent $\Delta F=2$ constraints that allow a large $s_{12}^{\lambda}$ only very close to the 12 -degeneracy line.

In figure 12 we show the allowed region of parameter space in the $\lambda_{1}-\lambda_{2}$-plane. As expected all parameter points lie very close to the exact 13-degeneracy line $\lambda_{2}=-2 \lambda_{1}$. 


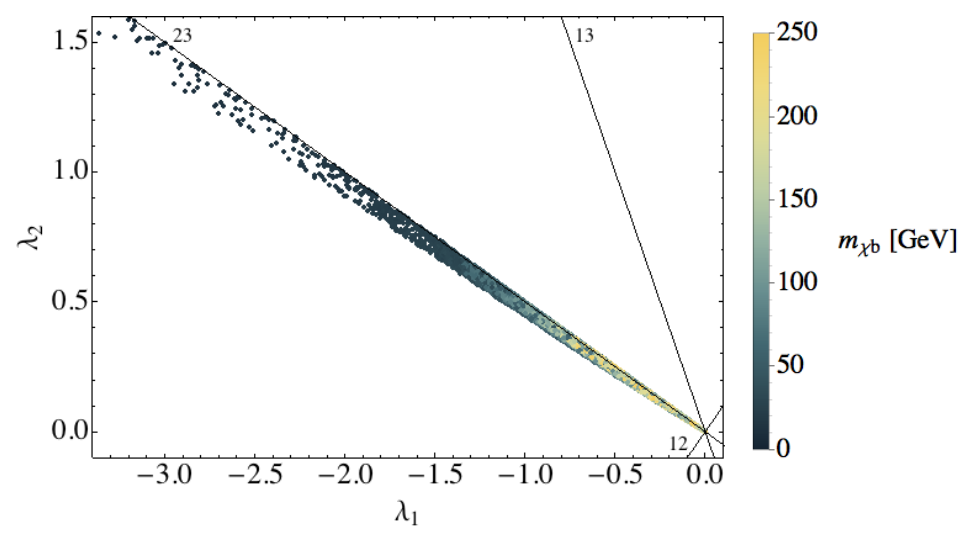

Figure 13. Allowed region in the $\lambda_{1}-\lambda_{2}$-plane for the 23-degeneracy scenario, after imposing the relic abundance, LUX and flavor constraints. The DM mass $m_{\chi_{b}}$ is indicated by the color, and the $i j$-degeneracy lines are sketched.

With decreasing DM mass $m_{\chi_{b}}$ they move further away from the universality point $\lambda_{1}=$ $\lambda_{2}=0$. This is a consequence of the required splitting of the second generation $m_{\chi_{s}}$.

\subsection{3-degeneracy}

The next scenario to study is the approximate 23-degeneracy, with $m_{\chi_{b}} \lesssim m_{\chi_{s}}<m_{\chi_{d}}$. In this case $s_{23}^{\lambda}$ is arbitrary, with $s_{12}^{\lambda}$ is required to be small by the $\Delta F=2$ constraints, and both $\Delta F=2$ and LUX constraints demand $s_{13}^{\lambda}$ to be small. In figure 13 we show the allowed parameter space for $\lambda_{1}$ and $\lambda_{2}$, which as expected is confined to be very close to the 23-degeneracy line $\lambda_{2}=-1 / 2 \lambda_{1}$. Again small DM mass $m_{\chi_{b}}$ implies a sizable coupling non-universality $\lambda_{1,2} \neq 0$. In contrast to the 13-degeneracy in this case no lower bound on $m_{\chi_{b}}$ emerges.

\subsection{Quasi-degenerate dark sector}

We finally study the case of a quasi-degenerate dark sector with mass splittings $<1 \%$. Such a scenario can be achieved either by having a quasi-universal coupling matrix $D_{\lambda}$ or by suppressing the DMFV correction to the mass matrix by the smallness of the parameter $\eta$. Note however that the correction is unavoidably generated at one loop level, so $|\eta| \lesssim 10^{-2}$ would involve fine-tuning. As in the previous cases we scan over the allowed parameter space for $\lambda$, assuming $b$-flavored DM and requiring the relic abundance to be set by thermal freeze-out. Since the three $\chi$ flavors are quasi-degenerate, all of them are present at freeze-out.

In figure 14 we show the allowed range of parameters in the $\lambda_{1}$ - $\lambda_{2}$-plane. The DM mass $m_{\chi_{b}}$ is varied and depicted by the color. We observe that for large DM masses $m_{\chi_{b}} \gtrsim 100 \mathrm{GeV}$ the allowed range of parameters is indeed close to the universality scenario $\lambda_{1,2}=0$. Some deviations are however allowed, as the non-universal corrections can be suppressed by a small expansion parameter $\eta$. For small DM masses however a significant deviation $\lambda_{1} \lesssim-1$ from the universality scenario is necessary and therefore $\eta$ has to be loop suppressed. A sizable negative $\lambda_{1}$, together with a near 23-degeneracy, suppresses the coupling $D_{\lambda, 11}$ with respect to the other $D_{\lambda}$ components. Consequently the relic abundance constraint is brought in accordance with the bound from LUX. 


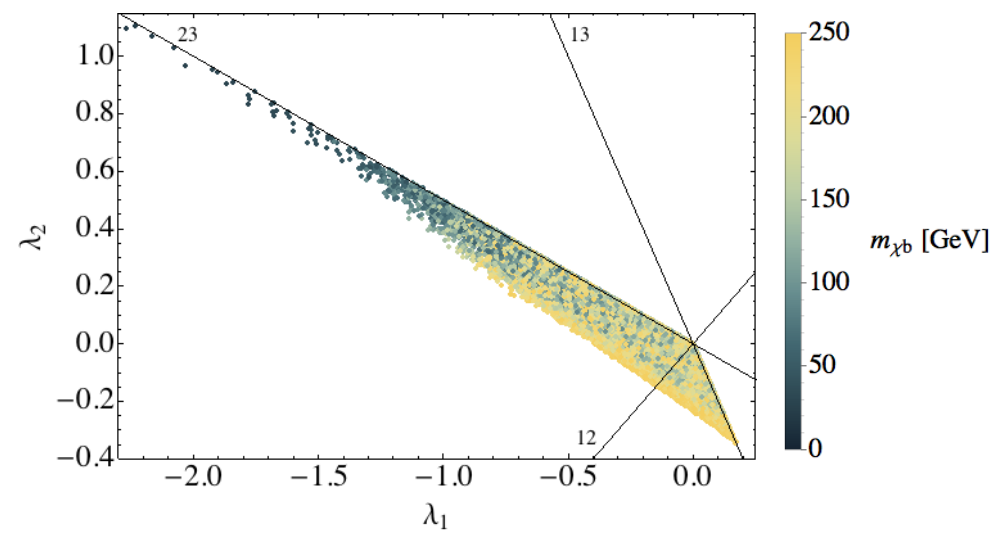

Figure 14. Allowed region in the $\lambda_{1}-\lambda_{2}$-plane for the quasi-degeneracy scenario, after imposing the relic abundance, LUX and flavor constraints. The DM mass $m_{\chi_{b}}$ is indicated by the color, and the $i j$-degeneracy lines are sketched.

\section{Collider phenomenology}

In this section we present the collider constraints on the mDMFV model. We expect significant constraints from the LHC since our model contains new colored states, as well as large coupling to quarks. Our framework predicts a number of new particles within the reach of the LHC:

- The scalar mediator $\phi$ which carries QCD charge and therefore has a large production cross-section in proton-proton collisions.

- The DM particle $\chi_{b}$ and the heavier flavors $\chi_{d, s}$, which are Dirac fermions and singlets under the SM gauge group.

This spectrum has some similarities to simplified models of squarks and neutralinos in the MSSM. The scalar mediator $\phi$ is similar to a right-handed down-type squark, except that it does not carry flavor. It couples to all three quark flavors $d, s, b$ with strengths given by the elements of the coupling matrix $\lambda$. The flavor is carried by $\chi$, leading to three somewhat degenerate states, in contrast to a neutralino LSP (lightest supersymmetric particle). Also note that in the simplest versions of supersymmetry neutralinos are Majorana fermions, whereas $\chi$ is a Dirac fermion.

A full treatment of collider constraints is beyond the scope of this work. We use existing analyses and translate their bounds into limits on our model. There are some qualitatively new features beyond the simplified MSSM model. First is the presence of large DM trilinear couplings with quarks. In the MSSM, supersymmetry constrains this coupling to be of the electroweak strength, making the production of squarks insensitive to the coupling with DM. In contrast, the large couplings required for relic abundance in the mDMFV model contribute non-negligibly to the production. This also makes the production sensitive to the flavor structure of the DM-quark couplings.

While large flavor angles in the mDMFV model are an interesting possibility, they lead to a large number of free parameters, and we postpone a detailed analysis of this case for 
future work. Here, we focus on the cases where the mixing angles are small. This has implications for both production and decay of $\phi$ and $\chi$.

The main constraints on the mDMFV model come from searches for monojets and dijets in conjunction with missing transverse energy. We now estimate what constraints these searches put on our model. We also point out some unexplored novel signatures that arise in this framework.

\subsection{Multijet with missing energy searches}

Let us begin with the constraints from multijet + MET searches. The relevant analyses that we consider for this case are the $19.5 \mathrm{fb}^{-1}$ CMS analysis for direct sbottom production [66] and multijet + MET analysis for squark-pair production [67]. The colored particle $\phi$ is analogous to a squark in the SUSY simplified models considered in these analyses, but with some important differences which we highlight below.

A dominant mode of $\phi$ pair production is through QCD interactions, in analogy with pair production of a single squark flavor, in the limit of decoupled gluino mass. There are two additional contributions that we need to consider. There is a sizeable contribution from the $t$-channel process mediated by $\chi_{d}$ due to the valence down quark pdf. Depending upon the value of the coupling $D_{\lambda, 11}$, this can be an $\mathcal{O}(1)$ fraction of the total production rate. The corresponding contribution from the $D_{\lambda, 22}$ is suppressed by the sea-quark $s$-quark pdf and does not contribute significantly.

There are also contributions from an off-shell quark $\left(g g \rightarrow d d^{*} \rightarrow d \chi_{d} \phi\right)$ which yield the same dijet + MET final state. The total production cross section for this process can be much higher than the QCD/t-channel production. However, this contribution always involves one jet arising from a gluon splitting, which typically carries a very small $p_{T}$. Thus, most events in this channel do not pass the selection cuts for dijet analysis.

As is familiar from squark pair-production, NLO QCD effects are significant. Since there is no NLO calculation available for our model, we present limits for two extreme cases: one is assuming the leading order production cross section, which provides a weak bound. The other limit is to take the squark production K-factor [68], and apply it to the LO cross section obtained above. Note that for the case $D_{\lambda, 11}=0$, the production cross section is given directly by the squark pair production cross section.

Another important difference from the sbottom case is the decay of the mediator $\phi$. In presence of small mixing, squarks decay into a flavor-specific jet and the LSP, whereas $\phi$ can decay to each of the quark flavors $d, s$ and $b$. This has the effect of weakening the limits relative to the sbottom search for sizeable branching fraction to the first and second generation. A similar effect has been observed for the case of stop scharm mixing in $[69,70]$, where the bounds on the stop mass get weakened due to the light jets in the final state.

In the CMS sbottom search [66], separate search regions are defined for one and two $b$-tags. For the SUSY simplified model, the two $b$-tag search region is the most sensitive for almost all of the parameter space. To translate limits from this analysis to the mDMFV case, we of course need to account for the branching fractions of $\phi$. The scaling is different for the one vs. two $b$-tag region, and it is possible that for some part of parameter space 

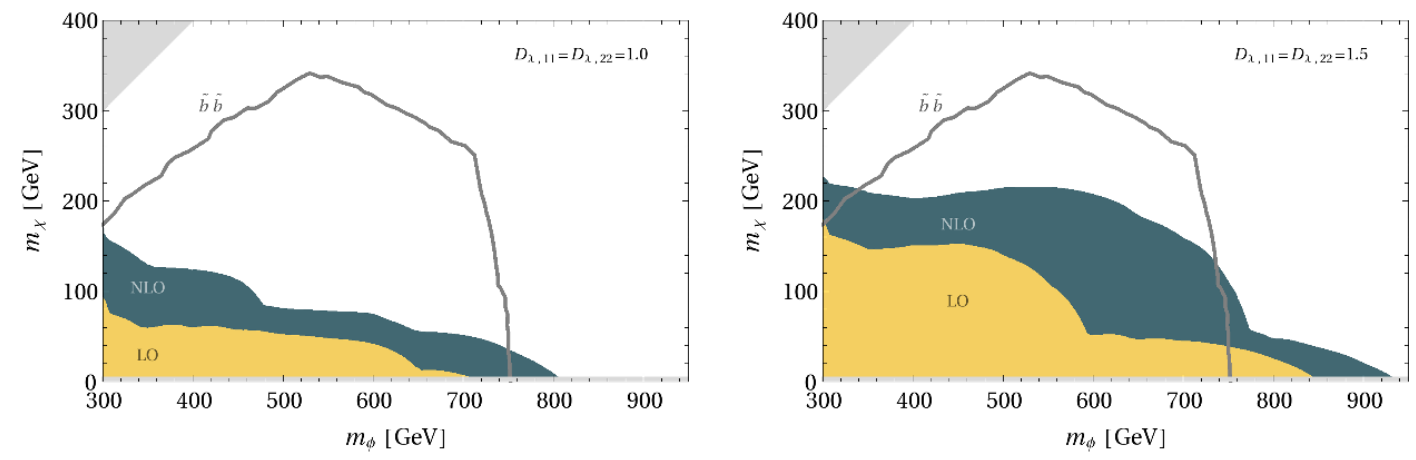

Figure 15. Limits on the mDMFV model from the CMS $19.5 \mathrm{fb}^{-1}$ sbottom [66] and squark [67] search. We show for comparison the CMS $\tilde{b} \tilde{b}$ limit (grey curve), which also directly applies to the case $D_{\lambda, 11}=D_{\lambda, 22}=0$. Shaded regions are excluded for particular choices of $D_{\lambda, 11}$ and $D_{\lambda, 22}$ as shown on the plot. For the NLO limit, we estimate the cross section by multiplying LO prediction with the NLO K-factor from sbottom pair production. The DM coupling to $b$-quark, $D_{\lambda, 33}$ is fixed everywhere by the corresponding relic abundance constraint, and all mixing angles are set to zero for simplicity.

the one $b$-tag region is more sensitive. For the current study we scale the cross section assuming the dominance of the two $b$-tag signal region.

There is no $b$-tag veto employed in the CMS squark search. Consequently, we can directly use the cross section limits obtained in that case. These limits are seen to be stronger than the sbottom limits for lower $m_{\phi}$ values, or for higher values of $D_{\lambda, 11}$. The latter is easily explained by the fact that in sbottom searches the increased production cross section for larger $D_{\lambda, 11}$ is offset by a smaller branching ratio into b-quarks.

We present our results in the $m_{\phi}-m_{\chi}$ mass plane in figure 15 . We choose three benchmark coupling values, $D_{\lambda, 11}=D_{\lambda, 22}=\{0,1,1.5\}$ to demonstrate the qualitative nature of the limits. The coupling $D_{\lambda, 33}$ is fixed to yield the correct relic abundance for each point on the plane, assuming a single flavor freeze-out. We show the limits from both the CMS $19.5 \mathrm{fb}^{-1}$ sbottom [66] and squark [67] searches. The NLO estimate is obtained from the LO cross section multiplied by K-factors from sbottom production. Larger $m_{\chi}$ correspond to a smaller $D_{\lambda, 33}$, and hence to a smaller $\phi \rightarrow b \chi_{b}$ branching ratio for fixed $D_{\lambda, 11}$ and $D_{\lambda, 22}$. This results in a much weaker limit for higher values of $m_{\chi}$ in figure 15 .

In figure 16, we present limits on $m_{\phi}$ for different values of $D_{\lambda, 11}$ and $D_{\lambda, 22}$, again with $D_{\lambda, 33}$ fixed by the relic abundance constraint. We choose a representative DM mass of $m_{\chi}=100 \mathrm{GeV}$. This plot shows the difference in sensitivity to the two different couplings - the constraint is much more severe for larger values of $D_{\lambda, 11}$ than for corresponding values of $D_{\lambda, 22}$ due to the enhanced production cross section in the former case.

\subsection{Monojet searches}

Another important constraint on the mDMFV parameter space is placed by the searches for monojets and large missing $E_{T}$. There are two interesting regimes for the monojet searches, the effective field theory (EFT) regime with a heavy mediator and the compressed spectrum regime. 


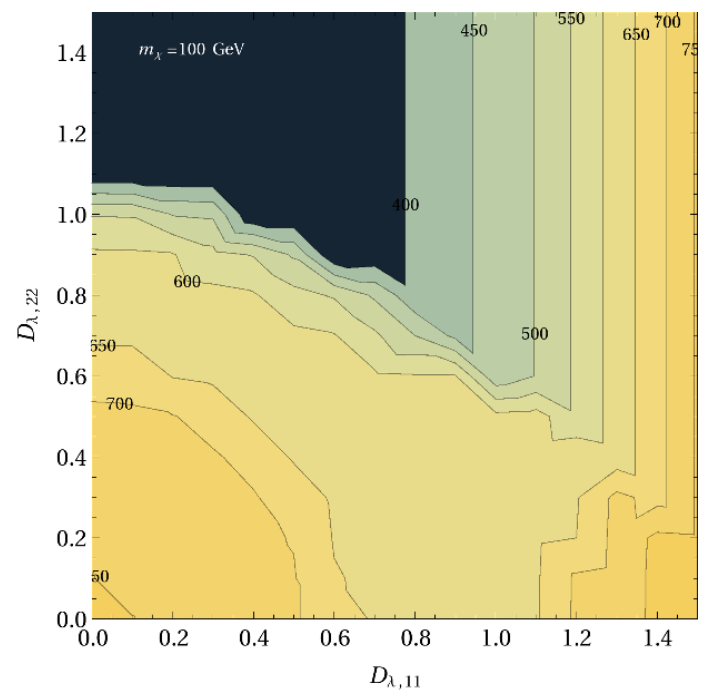

Figure 16. Exclusion contours for $m_{\phi}$ (in $\mathrm{GeV}$ ) for various values of $D_{\lambda, 11}$ and $D_{\lambda, 22}$ from the CMS sbottom [66] and squark [67] searches, obtained for the NLO estimate. $D_{\lambda, 33}$ is fixed by the relic abundance constraint.

For large mediator masses, the dominant signal arises from pair-production of DM along with a hard initial state radiation (ISR) jet. This can be crudely modeled by an EFT, which has the advantage of being largely model independent. Limits are presented as lower bounds on $\Lambda$, the scale associated with the coefficient of the higher dimensional operator [71]. However, exclusions using this technique have to be interpreted with care. In principle, the validity of the EFT fails for $\Lambda \lesssim p_{T}$, the $p_{T}$ cut employed in the searches. Thus, technically we can only exclude a band in $\Lambda$ self-consistently where the EFT is expected to be valid. CMS and ATLAS have put limits on various DM-quark EFT operators. The flavor specific operators that arise in our case have not been considered. However, we can estimate these limits by matching cross sections in our model with those from the DM EFT used by CMS/ATLAS.

In this case it is important to note that diagrams with an initial state $d$ quark are enhanced by the valence quark pdf. This process is therefore directly sensitive to the coupling $D_{\lambda, 11}$, suppressing that coupling would help to effectively evade the monojet constraints. In the DM mass regime we consider, $10 \mathrm{GeV}<m_{\chi}<250 \mathrm{GeV}$, the limits do not vary significantly. We show two benchmarks $m_{\chi}=10 \mathrm{GeV}$ and $m_{\chi}=210 \mathrm{GeV}$ as examples in figure 17.

The second regime in which the monojets provide a constraint complementary to the multijet searches is in the compressed region, $m_{\phi} \sim m_{\chi}$. In this region, the jets from $\phi$ decays are typically too soft to appear in multijet analyses. With a hard ISR jet, the signal resembles the DM monojet signal. The branching ratios of $\phi$ to different quark flavors are irrelevant in this case, since these decay products are typically too soft to pass the selection cuts. We show the limits obtained from recasting the CMS search for stops decaying to charm and a neutralino [72] in figure 18 . 

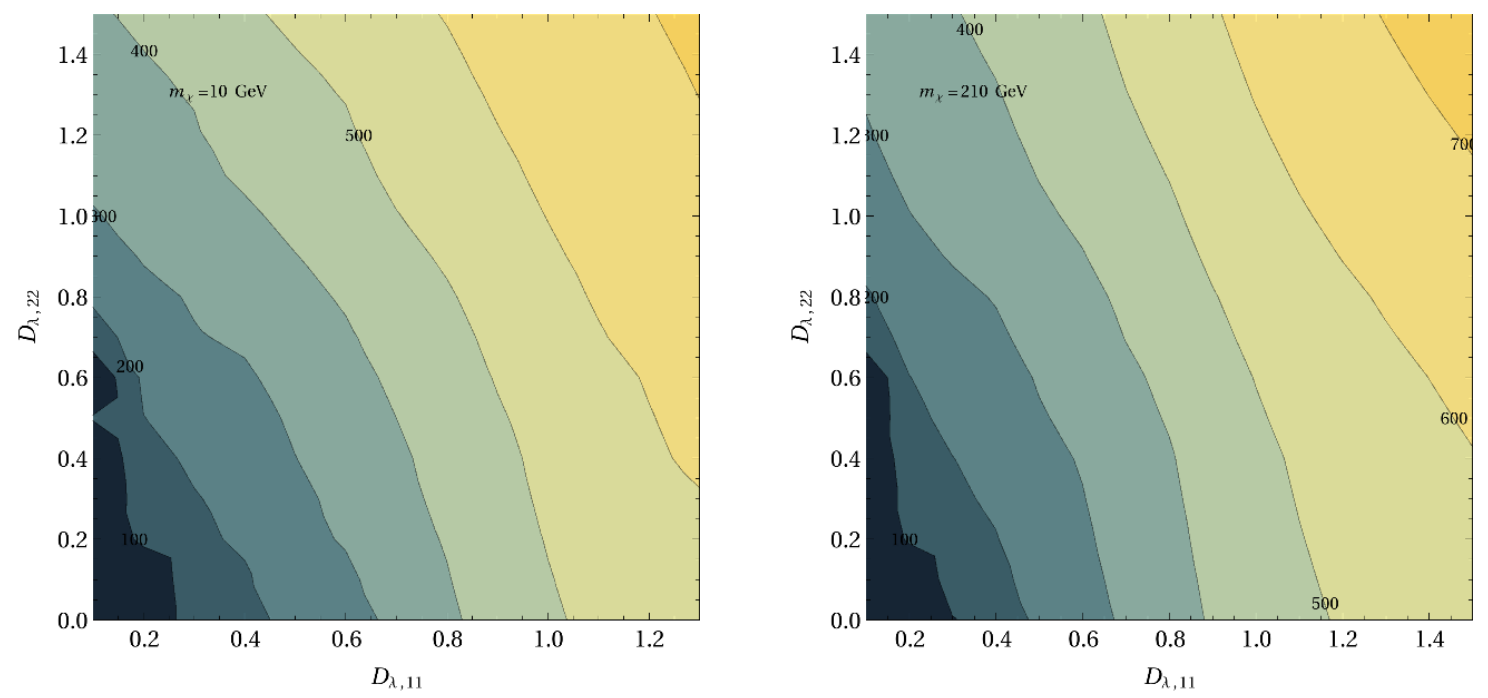

Figure 17. Limits on the mDMFV model in terms of mass of $\phi$ (in GeV) from the CMS monojet search [71]. Limits are obtained by matching production cross sections in our model with those in the DMEFT used by CMS.
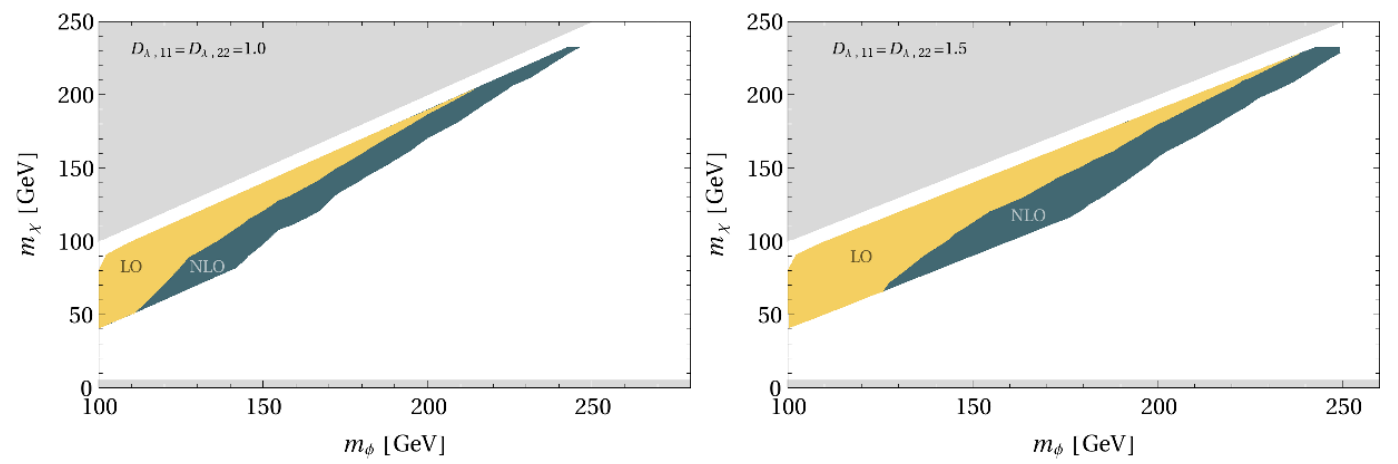

Figure 18. Limits on the mDMFV model from the CMS $19.7 \mathrm{fb}^{-1}$ search [72] for stops decaying to a charm and a neutralino, using the monojet + MET final state. The NLO cross section is estimated from the LO prediction with the NLO K-factor from sbottom pair production. The triangular grey regions are parts of parameter space where $\phi$ is lighter than the dark matter particles and hence cannot decay into them.

Since the monojet analyses typically veto additional jets, they lose sensitivity as the mass splitting between $\phi$ and $\chi$ increases. It has been pointed out that the razor analysis can be powerful in this region of parameter space [70, 73, 74]. It is an interesting future direction to analyze the constraints on this framework from razor.

A more quantitative analysis of the constraints on the masses of $\phi$ and $\chi_{i}$ would require a dedicated recasting of the experimental analyses which is beyond the scope of the present paper. 


\subsection{Distinctive signatures}

We see that a number of current searches are sensitive to this model. However, the presence of multiple flavors of DM have potentially novel signatures which could distinguish these models with other models of DM and colored mediators.

In the decay of $\phi$ all three DM flavors $\chi_{i}$ will be produced. While the lightest state $\chi_{b}$ is stable and simply escapes detection, the heavier states will decay to the lightest one through the decay channels discussed in appendix D.

In the case when the mass splitting between the $\chi_{i}$ are small, the decay products of the heavier states (jets, leptons or photons) can be too soft by themselves for the current searches to be sensitive. However, it is an interesting possibility to look for these relatively soft objects in conjunction with high $p_{T}$ jets. In the compressed regime, the heavier flavors can also have displaced decays, or decays in the detector.

When the mass splitting is larger, we expect a multijet signal, including heavy-flavor jets in addition to missing transverse momentum. Observation of these cascade decays would help in disentangling our model from other models of DM with colored mediators.

\section{Conclusions}

In this paper we presented a simplified model of flavored DM. The fermionic DM $\chi$ carries flavor and couples to right-handed down quarks transmitted by a colored scalar mediator $\phi$. We introduced the Dark Minimal Flavor Violation (DMFV) framework where the extended flavor symmetry $\mathrm{U}(3)_{q} \times \mathrm{U}(3)_{u} \times \mathrm{U}(3)_{d} \times \mathrm{U}(3)_{\chi}$ is broken only by the SM Yukawa couplings $Y_{u}, Y_{d}$ and the DM-quark coupling $\lambda$. In general the flavor violating effects generated by $\lambda$ can be large. Hence DMFV, while being conceptually similar to MFV, is phenomenologically very different from the latter scenario.

The DMFV assumption has the following implications that are very useful from a model building perspective:

- If DMFV is exact, an unbroken $\mathbb{Z}_{3}$ subgroup stabilizes the DM. It also prevents the new particles from being singly produced at the LHC.

- The DMFV hypothesis allows a reduction in the number of free parameters. The matrix $\lambda$ can be parametrized in terms of a unitary matrix $U_{\lambda}$ and a positive and diagonal matrix $D_{\lambda}$.

We performed a detailed phenomenological analysis of the minimal DMFV (mDMFV) model, focusing at first on the available constraints from flavor changing neutral current processes. Our findings can be summarized as follows:

- Meson antimeson mixing observables put strong constraints on the structure of the coupling matrix $\lambda$. Yet it is possible to satisfy all existing $\Delta F=2$ constraints simultaneously if $\lambda$ obeys one of the following scenarios:

1. Universality scenario - The diagonal coupling matrix $D_{\lambda}$ is nearly universal and large mixing angles in $U_{\lambda}$ are allowed. 
2. Small mixing scenario - The mixing matrix $U_{\lambda}$ is close to the unit matrix, while $D_{\lambda}$ is arbitrary.

3. $i j$-degeneracy scenarios $(i j=12,13,23)$ - Two elements of $D_{\lambda}$ are almost equal, $D_{\lambda, i i} \simeq D_{\lambda, j j}$, and only the corresponding mixing angle $s_{i j}^{\lambda}$ is allowed to be large.

Flavor violating effects are then efficiently suppressed by an almost diagonal matrix $\lambda \lambda^{\dagger}$. Our scenarios can straightforwardly be implemented in future studies. Note that their validity extends beyond the minimal model.

- The mDMFV model does not yield any new contribution to rare (semi-)leptonic $K$ and $B$ decays. ${ }^{3}$ Also the strongly constrained $B \rightarrow X_{s} \gamma$ decay does not receive any significant new contribution. Similarly new contributions to electroweak precision observables and electric dipole moments are small in mDMFV.

In the second step the constraints from DM phenomenology are included. We restricted our attention to the case of $b$-flavored DM which is phenomenologically most appealing. We also assumed that the observed DM relic density arises from thermal freeze-out. Our results are:

- The tree level contribution to DM-nucleon scattering, strongly constrained by the recent LUX data, needs to be suppressed by a small mixing angle $s_{13}^{\lambda}$.

- Further contributions to DM-nucleon scattering arise at the one-loop level from a box diagram and a photon penguin. These contributions become relevant for DM masses $m_{\chi_{b}} \lesssim 100 \mathrm{GeV}$. The penguin and box contributions carry a different overall sign, and hence destructively interfere if they are comparable in magnitude. The LUX constraint requires a partial cancellation, leading to both an upper and a lower bound on the size of $D_{\lambda, 11}$.

- We observe a non-trivial interplay of flavor and DM constraints, such that the combined constraint on the parameter space of $\lambda$ is an interesting overlap of the individual ones.

- We studied several mass spectra implying different freeze-out conditions. In all cases the penguin box cancellation implies a sizable splitting $D_{\lambda, 11}<D_{\lambda, 33}$ for DM masses $m_{\chi_{b}} \lesssim 100 \mathrm{GeV}$. This rules out the 13 -degeneracy scenario below this scale.

Stringent constraints on the mDMFV model also arise from direct searches at the LHC. We estimate the bounds arising from $b$-jets $+\mathbb{E}_{T}$ and monojet searches and point out possible new signatures. We find:

- Searches for direct sbottom quark production and for multiple jets plus missing energy constrain the mDMFV parameter space up to $m_{\phi} \simeq 800-900 \mathrm{GeV}$.

\footnotetext{
${ }^{3} \mathrm{~A}$ non-SM experimental signature for decays with neutrinos in the final state may arise if the DM particle is light enough that the decay into a $\chi \bar{\chi}$ final state is kinematically accessible [50].
} 
- Monojet searches are complementary to the searches for jets $+\mathbb{E}_{T}$ and competitive in two regions of parameter space, namely for large mediator masses or a compressed spectrum.

- The decay of the heavier $\chi$ flavors leads to additional jets or to soft photons, which could be looked for in conjunction with the monojet or dijet signals discussed above.

Our pioneering study of flavored DM with a generic flavor violating coupling matrix has revealed a lot of interesting phenomenology and showed that it is worthwhile to consider DM models beyond MFV.

\section{Acknowledgments}

PA and KG acknowledge partial support by the National Science Foundation under Grant No. PHYS-1066293 and the hospitality of the Aspen Center for Physics. KG was supported by the Deutsche Forschungsgemeinschaft (DFG), grant number GE 2541/1-1. Fermilab is operated by Fermi Research Alliance, LLC under Contract No. DE-AC02-07CH11359 with the United States Department of Energy.

\section{A Classification of DMFV symmetry structures}

In this appendix we classify the different possible versions of DMFV. A common feature is that the SM global flavor symmetry is extended by a $\mathrm{U}(3)_{\chi}$ in the dark sector. In addition to the SM Yukawa couplings there is only a single interaction $\lambda$ that breaks the flavor symmetry. In principle it is straightforward to implement DMFV in the lepton sector, but we restrict ourselves to the quark sector in what follows. The global flavor symmetry is then given by

$$
\mathrm{U}(3)_{q} \times \mathrm{U}(3)_{u} \times \mathrm{U}(3)_{d} \times \mathrm{U}(3)_{\chi} .
$$

Throughout this paper we assumed that the DM $\chi$ is a Dirac fermion and the mediator $\phi$ is a scalar, yet other Lorentz structures are also possible. We will not explore this option further.

In our analysis we considered the coupling of DM to the right-handed down type quarks by the structure

$$
\mathcal{L} \ni-\lambda \phi \bar{d}_{R} \chi_{L}
$$

In this case the coupling $\lambda$ induces the flavor symmetry breaking

$$
\mathrm{U}(3)_{d} \times \mathrm{U}(3)_{\chi} \rightarrow \mathrm{U}(1)_{d \oplus \chi}
$$

We classify this symmetry breaking pattern as $\mathrm{DMFV}_{d}$ in order to distinguish it from the following scenarios.

In $\mathrm{DMFV}_{u}$ instead DM couples to the right-handed up quarks,

$$
\mathcal{L} \ni-\lambda \phi \bar{u}_{R} \chi_{L}
$$


so that the symmetry breaking pattern is

$$
\mathrm{U}(3)_{u} \times \mathrm{U}(3)_{\chi} \rightarrow \mathrm{U}(1)_{u \oplus \chi} .
$$

Due to the coupling to up-type quarks, the phenomenology of $\mathrm{DMFV}_{u}$ is very different from the $\mathrm{DMFV}_{d}$ case. We leave a detailed study for future work.

Last but not least it is also possible to couple DM to the left-handed quark doublets

$$
\mathcal{L} \ni-\lambda \phi \bar{q}_{L} \chi_{R}
$$

In this case either $\phi$ or $\chi$ has to transform as an $\mathrm{SU}(2)_{L}$ doublet. In this so-called $\mathrm{DMFV}_{q}$ scenario, $\lambda$ yields the symmetry breaking

$$
\mathrm{U}(3)_{q} \times \mathrm{U}(3)_{\chi} \rightarrow \mathrm{U}(1)_{q \oplus \chi}
$$

The phenomenology of this scenario is enriched by the fact that now DM couples to both up and down type quarks.

\section{B $\quad \mathbb{Z}_{3}$ as an unbroken subgroup of DMFV}

In this appendix we present the proof for the presence of an unbroken $\mathbb{Z}_{3}$ subgroup emerging from the $\mathrm{SU}(3)_{\mathrm{QCD}} \times \mathrm{U}(3)_{q} \times \mathrm{U}(3)_{u} \times \mathrm{U}(3)_{d} \times \mathrm{U}(3)_{\chi}$ symmetry in the limit of exact DMFV, where $\chi$ transforms as a triplet under $\mathrm{U}(3)_{\chi}$. The argument is quite analogous to the one provided for the MFV case in [3]. The unbroken $\mathbb{Z}_{3}$ symmetry automatically forbids all operators inducing DM decay even at the non-renormalizable level, as long as they preserve DMFV. It also prevents the new particles from being singly produced at the LHC. Note that the validity of the proof does not depend on the Lorentz structure as it is based purely on internal symmetry arguments.

Let us consider the operator

$$
\mathcal{O} \sim \chi \ldots \bar{\chi} \ldots \phi \ldots \phi^{\dagger} \ldots q_{L} \ldots \bar{q}_{L} \ldots u_{R} \ldots \bar{u}_{R} \ldots d_{R} \ldots \bar{d}_{R} \ldots G \ldots \mathcal{S}
$$

where the dots represent an arbitrary number of insertions of the same fields, $G$ schematically denotes the gluon field or field strength and $S$ contains a combination of SM fields that are neutral under both QCD and the flavor symmetry. This is the most generic structure of interactions between the dark sector and SM particles. In the case $N_{\chi}=1, N_{\bar{\chi}}=$ $N_{\phi}=N_{\phi^{\dagger}}=0$ it mediates DM decay.

This structure is invariant under $\mathrm{QCD}$, if the number of $\mathrm{SU}(3)_{c}$ triplet minus the number of $\mathrm{SU}(3)_{c}$ anti-triplets is a multiple of three:

$$
\left(N_{\phi}-N_{\phi^{\dagger}}+N_{q}+N_{u}+N_{d}-N_{\bar{q}}-N_{\bar{u}}-N_{\bar{d}}\right) \quad \bmod 3=0 .
$$

Invariance of the operator $\mathcal{O}$ under the flavor symmetry can formally be achieved by treating the Yukawa couplings $Y_{u}, Y_{d}$ and $\lambda$ as spurion fields and adding a combination

$$
Y_{u} \ldots Y_{u}^{\dagger} \ldots Y_{d} \ldots Y_{d}^{\dagger} \ldots \lambda \ldots \lambda^{\dagger} \ldots
$$


to the operator $\mathcal{O}$. Invariance under $\mathrm{U}(3)_{q}$ then requires

$$
\left(N_{q}-N_{\bar{q}}+N_{Y_{u}}-N_{Y_{u}^{\dagger}}+N_{Y_{d}}-N_{Y_{d}^{\dagger}}\right) \quad \bmod 3=0 .
$$

Similarly $\mathrm{U}(3)_{u}, \mathrm{U}(3)_{d}$ and $\mathrm{U}(3)_{\chi}$ invariance give, respectively,

$$
\begin{aligned}
\left(N_{u}-N_{\bar{u}}-N_{Y_{u}}+N_{Y_{u}^{\dagger}}\right) & \bmod 3=0, \\
\left(N_{d}-N_{\bar{d}}-N_{Y_{d}}+N_{Y_{d}^{\dagger}}+N_{\lambda}-N_{\lambda^{\dagger}}\right) & \bmod 3=0, \\
\left(N_{\chi}-N_{\bar{\chi}}-N_{\lambda}+N_{\lambda^{\dagger}}\right) & \bmod 3=0 .
\end{aligned}
$$

Adding (B.4)-(B.7) and subtracting (B.2) we find

$$
\left(N_{\chi}-N_{\bar{\chi}}-N_{\phi}+N_{\phi^{\dagger}}\right) \quad \bmod 3=0
$$

as a necessary condition for the operator $\mathcal{O}$ to be invariant under QCD and the flavour symmetries. Eq. (B.8) can be interpreted as a $\mathbb{Z}_{3}$ symmetry under which $\chi$ and $\phi$ carry the charges $e^{+2 \pi i / 3}$ and $e^{-2 \pi i / 3}$, respectively, while the SM fields carry the charge +1 .

Consequently the combination of QCD and the DMFV assumption, together with our choice of representations forbid both $\chi$ and $\phi$ to decay into SM fields. Therefore in the exact DMFV limit the lightest state out of $\phi$ and the components of $\chi$ is stable without the need of imposing an additional symmetry.

\section{Relevant loop functions}

In this appendix we collect the one loop functions relevant for our analysis.

The NP one loop function for $\Delta F=2$ processes in mDMFV reads

$$
F\left(x_{i}, x_{j}\right)=\frac{x_{i}^{2} \log x_{i}}{\left(x_{i}-x_{j}\right)\left(1-x_{i}\right)^{2}}+\frac{x_{j}^{2} \log x_{j}}{\left(x_{j}-x_{i}\right)\left(1-x_{j}\right)^{2}}+\frac{1}{\left(1-x_{i}\right)\left(1-x_{j}\right)},
$$

where $x_{i}=m_{\chi_{i}}^{2} / m_{\phi}^{2}$. In the limit of degenerate masses $m_{\chi}$ it reduces to

$$
F(x)=\frac{2 x \log x}{(1-x)^{3}}+\frac{(1+x)}{(1-x)^{2}}
$$

with $x=m_{\chi}^{2} / m_{\phi}^{2}$.

The NP one loop contribution to the $b \rightarrow s \gamma$ transition is given by

$$
g(x)=\frac{1-5 x-2 x^{2}}{6(1-x)^{3}}-\frac{x^{2} \log x}{(1-x)^{4}}, \quad x_{i}=\frac{m_{\chi_{i}}^{2}}{m_{\phi}^{2}} .
$$

\section{Decay of heavier flavors}

DM flavors pick up mass splitting either through threshold corrections or through running. Consequently, the heavier $\chi$ flavors can decay into the lighter DM. The open decay modes depend both on the size of mass splittings as well as on the structure of the coupling matrix. 
As in the text, we focus on the case where $\chi_{b}$ is the lightest flavor, and hence constitutes the DM today. The relevant diagrams are shown in figure 19.

The dominant decay mode arises at tree-level, where the heavier DM decays into a pair of quarks and a lighter DM. This decay mode is only open if the splitting between the DM flavors is more than the lightest allowed mesons in the final state. Since this is a flavor-conserving decay, it is expected to dominate whenever allowed by phase space. Further, for couplings relevant for us, this decay mode is prompt on the timescale of BBN.

For more compressed spectra, the quark/meson final states may not be kinematically allowed. In this case, flavor-violating decays into a photon or a pair of leptons via an off-shell photon will be present. These can be estimated by using the effective coupling of DM particles with a photon $[4,75]$,

$$
\mathcal{L}_{\text {eff }}=\sum_{i=d, s, b} \frac{-\lambda_{i b}^{*} \lambda_{i h} e}{64 \pi^{2} m_{\phi}^{2}}\left[\left(\frac{1}{2}+\frac{2}{3} \log \left[\frac{m_{q_{i}}^{2}}{m_{\phi}^{2}}\right]\right) \mathcal{O}_{1, b h}+\frac{1}{4} \mathcal{O}_{2, b h}\right],
$$

where the subscript $h$ refers to the decaying heavier flavor of DM. The operators are give by

$$
\begin{aligned}
& \mathcal{O}_{1, b h}=\left[\bar{\chi}_{b} \gamma^{\mu}\left(1-\gamma^{5}\right) \partial^{\nu} \chi_{h}+\text { h.c. }\right] F_{\mu \nu}, \\
& \mathcal{O}_{2, b h}=\left[i \bar{\chi}_{b} \gamma^{\mu}\left(1-\gamma^{5}\right) \partial^{\nu} \chi_{h}+\text { h.c. }\right] F^{\sigma \rho} \epsilon_{\mu \nu \sigma \rho},
\end{aligned}
$$

and we have only kept the leading terms in momentum transfer, $\mathcal{O}\left(k^{2} / m_{\phi}^{2}\right)$, and in $m_{q} / m_{\phi}$.

We see immediately that operator $\mathcal{O}_{1}$ does not give rise to an on-shell photon decay, since the amplitude in that case is proportional to $k^{2}$. Similarly, the operator $\mathcal{O}_{2}$ contribution is seen to vanish because of a GIM-like cancellation. This is reminiscent of the decay $b \rightarrow s \gamma$ in the SM, where an analogous GIM cancellation causes the dominant decay to arise at a higher order in the EFT. We can then estimate the decay rate by the SM partonic $b \rightarrow s \gamma$ rate. We replace $G_{F}$ by the coefficient of the effective operator above, and account for an additional color factor. While the SM has spin-1 $W$ bosons running in the loop instead of the scalar $\phi$, this estimate should suffice for our purposes. The dominant contribution comes from the heaviest quark, i.e. the $b$-quark in the loop. The decay rate estimate is

$$
\begin{aligned}
\Gamma\left(\chi_{h} \rightarrow \chi_{b} \gamma\right) & \sim \frac{9\left|\lambda_{b h} \lambda_{b b}\right|^{2}}{2 \pi}\left[\frac{m_{b}^{2}}{m_{\phi}^{2}} \frac{e m_{\chi}}{\left(64 \pi^{2} m_{\phi}^{2}\right)}\right]^{2} \delta m^{3} \\
& \sim\left|\lambda_{b h} \lambda_{b b}\right|^{2}\left(\frac{1}{10^{5} \mathrm{sec}}\right)\left(\frac{1000 \mathrm{GeV}}{m_{\phi}}\right)^{8}\left(\frac{m_{\chi}}{10 \mathrm{GeV}}\right)^{2}\left(\frac{\delta m}{0.2 \mathrm{GeV}}\right)^{3}
\end{aligned}
$$

where $\delta m$ is the mass splitting between the heavy and the $b$-flavored DM. We see that this decay mode is too slow unless the dark matter is relatively heavy and the splitting is sizeable.

We now estimate the third contribution in figure 19. The operator $\mathcal{O}_{1}$ in equation (D.3) induces a four-fermion contact operator of the DM with SM leptons and light quarks. We can hence calculate the three-body decay rate in analogy with muon decay. The presence of the log prevents the GIM-like cancellation in this case. The estimate for the decay rate 

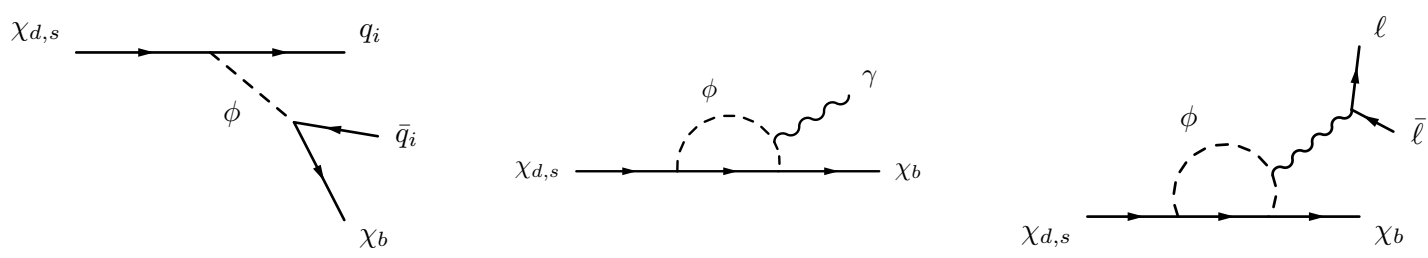

Figure 19. Decays of heavier flavors of DM into $\chi_{b}$.

to light SM fermions is given as,

$$
\begin{aligned}
\Gamma\left(\chi_{h} \rightarrow \chi_{b} f \bar{f}\right) & \sim\left|\lambda_{b h} \lambda_{b b}\right|^{2}\left[\frac{e^{2}}{96 \pi^{2} m_{\phi}^{2}} \log \left[\frac{m_{b}^{2}}{m_{q, h}^{2}}\right]\right]^{2} \frac{9 m^{5}}{192 \pi^{3}} \\
& \sim\left|\lambda_{b h} \lambda_{b b}\right|^{2}\left(\frac{1}{1 \mathrm{sec}}\right)\left(\frac{1000 \mathrm{GeV}}{m_{\phi}}\right)^{4}\left(\frac{\delta m}{0.2 \mathrm{GeV}}\right)^{5}
\end{aligned}
$$

where $m_{q, h}$ is the mass of the quark associated with the heavy flavor. We see that for relatively large values of the mass splitting, the DM decays well before the BBN epoch. On the edges of our parameter space, a more careful calculation is needed, especially in the region of small mixing angles.

Open Access. This article is distributed under the terms of the Creative Commons Attribution License (CC-BY 4.0), which permits any use, distribution and reproduction in any medium, provided the original author(s) and source are credited.

\section{References}

[1] J. Kile and A. Soni, Flavored Dark Matter in Direct Detection Experiments and at LHC, Phys. Rev. D 84 (2011) 035016 [arXiv: 1104.5239] [INSPIRE].

[2] J.F. Kamenik and J. Zupan, Discovering Dark Matter Through Flavor Violation at the LHC, Phys. Rev. D 84 (2011) 111502 [arXiv:1107.0623] [InSPIRE].

[3] B. Batell, J. Pradler and M. Spannowsky, Dark Matter from Minimal Flavor Violation, JHEP 08 (2011) 038 [arXiv: 1105.1781] [InSPIRE].

[4] P. Agrawal, S. Blanchet, Z. Chacko and C. Kilic, Flavored Dark Matter and Its Implications for Direct Detection and Colliders, Phys. Rev. D 86 (2012) 055002 [arXiv:1109.3516] [INSPIRE].

[5] B. Batell, T. Lin and L.-T. Wang, Flavored Dark Matter and R-Parity Violation, JHEP 01 (2014) 075 [arXiv: 1309.4462] [INSPIRE].

[6] J. Kile, Flavored Dark Matter: A Review, Mod. Phys. Lett. A 28 (2013) 1330031 [arXiv: 1308.0584] [INSPIRE].

[7] L. Lopez-Honorez and L. Merlo, Dark matter within the minimal flavour violation ansatz, Phys. Lett. B 722 (2013) 135 [arXiv:1303.1087] [INSPIRE].

[8] A. Kumar and S. Tulin, Top-flavored dark matter and the forward-backward asymmetry, Phys. Rev. D 87 (2013) 095006 [arXiv:1303.0332] [INSPIRE]. 
[9] Y. Zhang, Top Quark Mediated Dark Matter, Phys. Lett. B 720 (2013) 137 [arXiv:1212.2730] [INSPIRE].

[10] M. Hirsch, S. Morisi, E. Peinado and J.W.F. Valle, Discrete dark matter, Phys. Rev. D 82 (2010) 116003 [arXiv: 1007.0871] [INSPIRE].

[11] M.S. Boucenna et al., Phenomenology of Dark Matter from $A_{4}$ Flavor Symmetry, JHEP 05 (2011) 037 [arXiv:1101.2874] [INSPIRE].

[12] L.E. Ibáñez, The Scalar Neutrinos as the Lightest Supersymmetric Particles and Cosmology, Phys. Lett. B 137 (1984) 160 [inSPIRE].

[13] J.R. Ellis, J.S. Hagelin, D.V. Nanopoulos, K.A. Olive and M. Srednicki, Supersymmetric Relics from the Big Bang, Nucl. Phys. B 238 (1984) 453 [InSPIRE].

[14] J.S. Hagelin, G.L. Kane and S. Raby, Perhaps Scalar Neutrinos Are the Lightest Supersymmetric Partners, Nucl. Phys. B 241 (1984) 638 [InSPIRE].

[15] M.W. Goodman and E. Witten, Detectability of Certain Dark Matter Candidates, Phys. Rev. D 31 (1985) 3059 [INSPIRE].

[16] K. Freese, Can Scalar Neutrinos Or Massive Dirac Neutrinos Be the Missing Mass?, Phys. Lett. B 167 (1986) 295 [INSPIRE].

[17] T. Falk, K.A. Olive and M. Srednicki, Heavy sneutrinos as dark matter, Phys. Lett. B 339 (1994) 248 [hep-ph/9409270] [INSPIRE].

[18] J. March-Russell, C. McCabe and M. McCullough, Neutrino-Flavoured Sneutrino Dark Matter, JHEP 03 (2010) 108 [arXiv:0911.4489] [INSPIRE].

[19] T. Daylan et al., The Characterization of the Gamma-Ray Signal from the Central Milky Way: A Compelling Case for Annihilating Dark Matter, arXiv:1402.6703 [INSPIRE].

[20] A. Berlin, D. Hooper and S.D. McDermott, Simplified Dark Matter Models for the Galactic Center Gamma-Ray Excess, Phys. Rev. D 89 (2014) 115022 [arXiv:1404.0022] [INSPIRE].

[21] P. Agrawal, B. Batell, D. Hooper and T. Lin, Flavored Dark Matter and the Galactic Center Gamma-Ray Excess, Phys. Rev. D 90 (2014) 063512 [arXiv:1404.1373] [InSPIRE].

[22] E. Izaguirre, G. Krnjaic and B. Shuve, The Galactic Center Excess from the Bottom Up, Phys. Rev. D 90 (2014) 055002 [arXiv:1404.2018] [INSPIRE].

[23] C. Boehm, M.J. Dolan, C. McCabe, M. Spannowsky and C.J. Wallace, Extended gamma-ray emission from Coy Dark Matter, JCAP 05 (2014) 009 [arXiv:1401.6458] [INSPIRE].

[24] S. Ipek, D. McKeen and A.E. Nelson, A Renormalizable Model for the Galactic Center Gamma Ray Excess from Dark Matter Annihilation, Phys. Rev. D 90 (2014) 055021 [arXiv: 1404.3716] [INSPIRE].

[25] K. Kong and J.-C. Park, Bounds on Dark Matter Interpretation of Fermi-LAT GeV Excess, arXiv: 1404.3741 [INSPIRE].

[26] P. Ko, W.-I. Park and Y. Tang, Higgs portal vector dark matter for $\mathrm{GeV}$ scale $\gamma$-ray excess from galactic center, JCAP 09 (2014) 013 [arXiv: 1404.5257] [INSPIRE].

[27] C. Boehm, M.J. Dolan and C. McCabe, A weighty interpretation of the Galactic Centre excess, Phys. Rev. D 90 (2014) 023531 [arXiv:1404.4977] [inSPIRE].

[28] M. Abdullah et al., Hidden On-Shell Mediators for the Galactic Center Gamma-Ray Excess, Phys. Rev. D 90 (2014) 035004 [arXiv: 1404.6528] [INSPIRE]. 
[29] D.K. Ghosh, S. Mondal and I. Saha, Confronting the Galactic Center Gamma Ray Excess With a Light Scalar Dark Matter, arXiv:1405.0206 [INSPIRE].

[30] A. Martin, J. Shelton and J. Unwin, Fitting the Galactic Center Gamma-Ray Excess with Cascade Annihilations, arXiv:1405.0272 [INSPIRE].

[31] A. Berlin, P. Gratia, D. Hooper and S.D. McDermott, Hidden Sector Dark Matter Models for the Galactic Center Gamma-Ray Excess, Phys. Rev. D 90 (2014) 015032 [arXiv:1405.5204] [INSPIRE].

[32] T. Basak and T. Mondal, Class of Higgs-portal Dark Matter models in the light of gamma-ray excess from Galactic center, arXiv:1405.4877 [INSPIRE].

[33] K.P. Modak, D. Majumdar and S. Rakshit, A Possible Explanation of Low Energy $\gamma$-ray Excess from Galactic Centre and Fermi Bubble by a Dark Matter Model with Two Real Scalars, arXiv: 1312.7488 [INSPIRE].

[34] R.S. Chivukula and H. Georgi, Composite Technicolor Standard Model, Phys. Lett. B 188 (1987) 99 [INSPIRE].

[35] L.J. Hall and L. Randall, Weak scale effective supersymmetry, Phys. Rev. Lett. 65 (1990) 2939 [INSPIRE].

[36] A.J. Buras, P. Gambino, M. Gorbahn, S. Jager and L. Silvestrini, Universal unitarity triangle and physics beyond the standard model, Phys. Lett. B 500 (2001) 161 [hep-ph/0007085] [INSPIRE].

[37] G. D'Ambrosio, G.F. Giudice, G. Isidori and A. Strumia, Minimal flavor violation: An Effective field theory approach, Nucl. Phys. B 645 (2002) 155 [hep-ph/0207036] [INSPIRE].

[38] A.J. Buras, Minimal flavor violation, Acta Phys. Polon. B 34 (2003) 5615 [hep-ph/0310208] [INSPIRE].

[39] LUX collaboration, D.S. Akerib et al., First results from the LUX dark matter experiment at the Sanford Underground Research Facility, Phys. Rev. Lett. 112 (2014) 091303 [arXiv: 1310.8214] [INSPIRE].

[40] J.L. Feng, J. Kumar, D. Marfatia and D. Sanford, Isospin-Violating Dark Matter, Phys. Lett. B 703 (2011) 124 [arXiv: 1102.4331] [INSPIRE].

[41] J.L. Feng, J. Kumar and D. Sanford, Xenophobic Dark Matter, Phys. Rev. D 88 (2013) 015021 [arXiv: 1306.2315] [INSPIRE].

[42] M. Blanke et al., Another look at the flavour structure of the littlest Higgs model with T-parity, Phys. Lett. B 646 (2007) 253 [hep-ph/0609284] [INSPIRE].

[43] M. Blanke, A.J. Buras, K. Gemmler and T. Heidsieck, $\Delta F=2$ observables and $B \rightarrow X_{q} \gamma$ decays in the Left-Right Model: Higgs particles striking back, JHEP 03 (2012) 024 [arXiv:1111.5014] [INSPIRE].

[44] A.J. Buras and J. Girrbach, Towards the Identification of New Physics through Quark Flavour Violating Processes, Rept. Prog. Phys. 77 (2014) 086201 [arXiv:1306.3775] [INSPIRE].

[45] A.J. Buras, F. De Fazio, J. Girrbach and M.V. Carlucci, The Anatomy of Quark Flavour Observables in 331 Models in the Flavour Precision Era, JHEP 02 (2013) 023 [arXiv: 1211.1237] [INSPIRE]. 
[46] J. Virto, Exact NLO strong interaction corrections to the $\Delta F=2$ effective Hamiltonian in the MSSM, JHEP 11 (2009) 055 [arXiv:0907.5376] [INSPIRE].

[47] S. Bertolini, F. Borzumati, A. Masiero and G. Ridolfi, Effects of supergravity induced electroweak breaking on rare B decays and mixings, Nucl. Phys. B 353 (1991) 591 [InSPIRE].

[48] P.L. Cho, M. Misiak and D. Wyler, $K_{L} \rightarrow \pi^{0} e^{+} e^{-}$and $B \rightarrow X_{s} l^{+} l^{-}$decay in the MSSM, Phys. Rev. D 54 (1996) 3329 [hep-ph/9601360] [INSPIRE].

[49] W. Altmannshofer and D.M. Straub, New physics in $B \rightarrow K^{*} \mu \mu$ ?, Eur. Phys. J. C 73 (2013) 2646 [arXiv: 1308.1501] [INSPIRE].

[50] J.F. Kamenik and C. Smith, FCNC portals to the dark sector, JHEP 03 (2012) 090 [arXiv:1111.6402] [INSPIRE].

[51] S.M. Barr and A. Zee, Electric Dipole Moment of the Electron and of the Neutron, Phys. Rev. Lett. 65 (1990) 21 [Erratum ibid. 65 (1990) 2920] [INSPIRE].

[52] UTFIT collaboration, M. Bona et al., The UTfit collaboration report on the status of the unitarity triangle beyond the standard model. I. Model-independent analysis and minimal flavor violation, JHEP 03 (2006) 080 [hep-ph/0509219] [INSPIRE]. Updates available on http://www.utfit.org.

[53] UTfit collaboration, M. Bona et al., Model-independent constraints on $\Delta F=2$ operators and the scale of new physics, JHEP 03 (2008) 049 [arXiv:0707.0636] [INSPIRE].

[54] A.J. Buras, F. De Fazio and J. Girrbach, 331 models facing new $b \rightarrow s \mu^{+} \mu^{-}$data, JHEP 02 (2014) 112 [arXiv:1311.6729] [INSPIRE].

[55] J. Hubisz, S.J. Lee and G. Paz, The Flavor of a little Higgs with T-parity, JHEP 06 (2006) 041 [hep-ph/0512169] [INSPIRE].

[56] M. Blanke et al., Particle antiparticle mixing, $\varepsilon_{K}, \Delta \Gamma_{q}, A_{S L}^{q}, A_{C P}\left(B_{d} \rightarrow \psi K_{S}\right)$, $A_{C P}\left(B_{s} \rightarrow \psi \phi\right)$ and $B \rightarrow X_{s, d} \gamma$ in the Littlest Higgs model with T-parity, JHEP 12 (2006) 003 [hep-ph/0605214] [INSPIRE].

[57] M. Blanke, A.J. Buras, B. Duling, S. Recksiegel and C. Tarantino, FCNC Processes in the Littlest Higgs Model with T-Parity: a 2009 Look, Acta Phys. Polon. B 41 (2010) 657 [arXiv:0906.5454] [INSPIRE].

[58] C. Csáki, A. Falkowski and A. Weiler, The Flavor of the Composite Pseudo-Goldstone Higgs, JHEP 09 (2008) 008 [arXiv:0804.1954] [INSPIRE].

[59] M. Blanke, A.J. Buras, B. Duling, S. Gori and A. Weiler, $\Delta F=2$ Observables and Fine-Tuning in a Warped Extra Dimension with Custodial Protection, JHEP 03 (2009) 001 [arXiv: 0809.1073] [INSPIRE].

[60] M. Bauer, S. Casagrande, U. Haisch and M. Neubert, Flavor Physics in the Randall-Sundrum Model: II. Tree-Level Weak-Interaction Processes, JHEP 09 (2010) 017 [arXiv: 0912.1625] [INSPIRE].

[61] Y. Zhang, H. An, X. Ji and R.N. Mohapatra, General CP-violation in Minimal Left-Right Symmetric Model and Constraints on the Right-Handed Scale, Nucl. Phys. B 802 (2008) 247 [arXiv: 0712.4218] [INSPIRE].

[62] G. Isidori, Y. Nir and G. Perez, Flavor Physics Constraints for Physics Beyond the Standard Model, Ann. Rev. Nucl. Part. Sci. 60 (2010) 355 [arXiv:1002.0900] [InSPIRE]. 
[63] K. Griest and D. Seckel, Three exceptions in the calculation of relic abundances, Phys. Rev. D 43 (1991) 3191 [INSPIRE].

[64] G. Servant and T.M.P. Tait, Is the lightest Kaluza-Klein particle a viable dark matter candidate?, Nucl. Phys. B 650 (2003) 391 [hep-ph/0206071] [INSPIRE].

[65] G. Steigman, B. Dasgupta and J.F. Beacom, Precise Relic WIMP Abundance and its Impact on Searches for Dark Matter Annihilation, Phys. Rev. D 86 (2012) 023506 [arXiv: 1204.3622] [INSPIRE].

[66] CMS collaboration, Search for direct production of bottom squark pairs, CMS-PAS-SUS-13-018 (2014).

[67] CMS collaboration, Search for new physics in the multijet and missing transverse momentum final state in proton-proton collisions at $\sqrt{s}=8 \mathrm{TeV}$, JHEP 06 (2014) 055 [arXiv: 1402.4770] [INSPIRE].

[68] M. Krämer et al., Supersymmetry production cross sections in pp collisions at $\sqrt{s}=7 \mathrm{TeV}$, arXiv:1206. 2892 [INSPIRE].

[69] M. Blanke, G.F. Giudice, P. Paradisi, G. Perez and J. Zupan, Flavoured Naturalness, JHEP 06 (2013) 022 [arXiv: 1302.7232] [INSPIRE].

[70] P. Agrawal and C. Frugiuele, Mixing stops at the LHC, JHEP 01 (2014) 115 [arXiv: 1304.3068] [INSPIRE].

[71] CMS collaboration, Search for new physics in monojet events in pp collisions at $\sqrt{s}=8 \mathrm{TeV}$, CMS-PAS-EXO-12-048 (2013).

[72] CMS collaboration, Search for top squarks decaying to a charm quark and a neutralino in events with a jet and missing transverse momentum, CMS-PAS-SUS-13-009 (2014).

[73] P.J. Fox, R. Harnik, R. Primulando and C.-T. Yu, Taking a Razor to Dark Matter Parameter Space at the LHC, Phys. Rev. D 86 (2012) 015010 [arXiv:1203.1662] [INSPIRE].

[74] CMS collaboration, Search for supersymmetry with razor variables in pp collisions at $\sqrt{s}=7 \mathrm{TeV}$, arXiv:1405.3961 [INSPIRE].

[75] J. Kopp, V. Niro, T. Schwetz and J. Zupan, DAMA/LIBRA and leptonically interacting Dark Matter, Phys. Rev. D 80 (2009) 083502 [arXiv:0907.3159] [INSPIRE]. 